\title{
Polylactide Single-Polymer Composites with a Wide Melt- Processing Window Based on Core-Sheath PLA Fibers
}

DOI:

10.1016/j.matdes.2017.10.066

Document Version

Accepted author manuscript

Link to publication record in Manchester Research Explorer

\section{Citation for published version (APA):}

Liu, Q., Zhao, M., Zhou, Y., Yang, Q., Shen, Y., Gong, R., Zhou, F., Li, Y., \& Deng, B. (2018). Polylactide SinglePolymer Composites with a Wide Melt-Processing Window Based on Core-Sheath PLA Fibers. Materials \& Design, 139, 36-44. https://doi.org/10.1016/j.matdes.2017.10.066

\section{Published in:}

Materials \& Design

\section{Citing this paper}

Please note that where the full-text provided on Manchester Research Explorer is the Author Accepted Manuscript or Proof version this may differ from the final Published version. If citing, it is advised that you check and use the publisher's definitive version.

\section{General rights}

Copyright and moral rights for the publications made accessible in the Research Explorer are retained by the authors and/or other copyright owners and it is a condition of accessing publications that users recognise and abide by the legal requirements associated with these rights.

\section{Takedown policy}

If you believe that this document breaches copyright please refer to the University of Manchester's Takedown Procedures [http://man.ac.uk/04Y6Bo] or contact uml.scholarlycommunications@manchester.ac.uk providing relevant details, so we can investigate your claim.

\section{OPEN ACCESS}




\title{
Attitude controller design for reusable launch vehicles during reentry phase via compound adaptive fuzzy H-infinity control
}

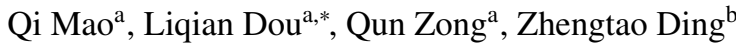 \\ ${ }^{a}$ School of Electrical and Information Engineering, Tianjin University Tianjin, 300072, China \\ ${ }^{b}$ School of Electrical and Electronic Engineering, The University of Manchester, Manchester, M13 9PL, U.K.
}

\begin{abstract}
In this paper, the attitude control problem of reusable launch vehicles (RLVs) during reentry phase is investigated by using compound adaptive fuzzy H-infinity control (CAFHC) strategy in the presence of parameter uncertainties and external disturbances. Firstly, the control-oriented attitude model is established by a model transformation based on the six-degree-of-freedom(6-DoF) dynamic model of the RLV. Secondly, a novel attitude control scheme is developed and the control strategy consists of two parts to achieve a stable and accurate attitude tracking during reentry flight process. An attitude tracking controller is designed utilizing adaptive fuzzy $\mathrm{H}$-infinity control approach combined with an identification model to improve the attitude tracking performance in the interior of fuzzy approximation region of attitude angle. Next, an attitude stabilization controller based on boundary adaptive technique is employed to assure the robustness of the closed-loop system in the exterior of fuzzy approximation region of attitude angle. Furthermore, the stability of the closed-loop system is guaranteed within the framework of Lyapunov theory and the attitude tracking error converges to a small neighborhood around origin. Finally, the simulation results are presented to demonstrate that the effectiveness of the proposed control scheme for reentry RLV, and its tracking performance performs better than the other control method.
\end{abstract}

Keywords: reusable launch vehicle; attitude control; fuzzy logic system; H-infinity control; reentry phase

\section{Introduction}

With the aim to develop a more cost-effective and reliable 25 approach to the space, reusable launch vehicles (RLVs) have attracted intensive research interest in the field of aerospace 5 engineering[1][2][3]. Mainly due to the unique advantages in reusability, flexibility as well as low operational cost, RLVs have a good prospect in aerospace activities and broad applica- 30 tions in civilian and military fields [4]. Whereas the philosophy sounds interesting and attracting, a major challenging problem 10 posed in flight missions is that of atmospheric reentry. During the reentry phase, the altitude and velocity of the vehicle vary rapidly and drastically as a RLV goes through a wide range of 35 flight envelope. At the same time, RLVs are subject to the poor flight conditions, severe parameter uncertainties and unknown external disturbances [5][6][7]. Thus, the reentry attitude control of RLVs is still a challenging problem [8], and it is critical for the RLV to track the expected commands rapidly and accu- 40 rately.

In the recent past, a variety of control approaches have been proposed for designing the flight controllers of the vehicle. In the early studies, the flight control problem was investigated based on a linearized model of aerocrafts [9][10], whereas it 45

\footnotetext{
${ }^{*}$ Corresponding author: Liqian Dou

Email addresses: maoqi1118@t ju.edu.cn (Qi Mao), douliqian@tju.edu.com (Liqian Dou ), zongqun@tju.edu.cn (Qun Zong), zhengtao.ding@manchester.ac.uk (Zhengtao Ding)
}

is unsuitable for highly nonlinear and multivariable model of the RLV. Then, gain scheduling (GS) [11][12][13] as a popular control approach was applied for flight control system design. Although gain scheduling control method is verified to be effective to solve some control issues for reentry vehicles, the system robustness and global stability cannot be guaranteed especially under the circumstance of abrupt change of the control parameters. In the work of [14], model predictive control (MPC) method was combined with feedback linearization to develop a controller for reentry vehicle. After that, the trajectory linearization control (TLC) [15][16][17] scheme was developed to improve the nonlinear performance of GS in flight control system for aircrafts. But the robust performance of this approach is finite as the tracking dynamics are linearized by the trajectory linearization controller. Analogously, dynamic inversion (DI) technique [18][19][20] was proposed to cope with the flight control problem of a reusable launch vehicle. However, the drawback of this strategy is poor in robustness for parameter uncertainties and modeling errors if improperly designed. Further, Lam and Krishnamurthy et.al. have investigated an adaptive controller using the state dependent Riccati equation (SDRE) approach [21] to a twin-rotor aircraft, but it is difficult for SDRE to deal with the control system with a high order. In [22][23], backstepping methodology was implemented to design the flight control system of the vehicle. Nevertheless, it is worth noting that the external disturbance was not taken into consideration in [22]-[23].

For the purpose of designing an attitude controller with a 
good robust performance, Shtessel et.al. [24][25] have explored the application of sliding mode control (SMC) scheme to attitude control system of RLVs. SMC technique is well known $n_{110}$ as a robust method to design control law for uncertain system.

55 However, the boundary values of model uncertainties and external disturbances ought to be known a prior and it is hard to satisfy this requirement in practical experiment. In [26][27], robust control approach was used to tackle the flight control $_{115}$ issue in the presence of hard constrains, model uncertainties and external disturbances for RLVs. Similarly, Jee and Yalagach et.al. have addressed H-infinity control strategy [28] to design flight control system under the highly uncertainties and changing dynamics of aerodynamic coefficients for the RLV during reentry process. However, robust control scheme cannot achieve a good tracking performance easily since the robust controller is designed in the worst case of the control system. And then, adaptive control [29][30] technique was employed to combine with robust control method in order to compensate for its shortcomings and improve the system tracking perfor70 mance. Recently, fuzzy logic system (FLS) [31][32][33][34] was applied to design the attitude controller of reentry vehicle for its good approximation to model uncertainties and un-125 modeled dynamics. This control approach is an efficient way to cope with the model uncertainties and external disturbances 75 even if the boundaries of uncertainties and disturbances are unknown. Moreover, FLS are always combined with other control strategies, such as adaptive control [35], robust control [36], fault-tolerant control [37] and so on, with the goal to improve the tracking control performance of the flight control system and achieve a good robustness simultaneously. Besides, adaptive fuzzy control method is often combined with robust or $\mathrm{H}$ infinity control method in many applications [38][39][40].

In this research, we will further focus on the attitude controller design problem of RLVs during its reentry phase where parameter uncertainties and external disturbances are considered, and propose a compound adaptive fuzzy $\mathrm{H}$-infinity control (CAFHC) strategy. Wherein, the fuzzy logic system is introduced to approximate the uncertainty term, and the H-infinity controller is adopted to compensate for fuzzy modeling errors 90 and the external disturbances. However, the introduction of $\mathrm{H}$ infinity control term would degrade the approximation capability of FLS, which would further weaken the tracking perfor- ${ }^{130}$ mance of reentry RLV. In order to avoid the problem of "approximation capability weakening", a novel control scheme is 95 developed and this control strategy mainly consists of two parts. An attitude tracking controller is designed utilizing adaptive fuzzy $\mathrm{H}$-infinity control approach combined with an identification model to improve the attitude tracking performance, while an attitude stabilization controller based on boundary adaptive

100 technique is employed to assure the system robustness and the boundedness of approximation error. Moreover, the stability analysis is carried out to demonstrate that the proposed strategy can guarantee the semi-global stability of the closed-loop system. Finally, the simulation results of 6-DoF dynamic mode 105 for the RLV are presented to illustrate the effectiveness of the proposed control strategy.

The rest of this paper is organized as follows. The 6-DoF dynamic model and the control-oriented attitude model of reentry RLV are stated in Section 2. Next the attitude controller design strategy via CAFHC approach is developed, followed by the stability analysis of the closed-loop control system in Section 3. After that the numerical simulations applying the proposed control scheme are conducted, and the simulation results and discussions are presented in Section 4. Finally, the conclusion of the paper is drawn in Section 5.

\section{Problem formulation}

In this section, the 6-DoF dynamic model of the RLV within its reentry phase is described, and the control-oriented attitude model is derived by a model transformation to design the attitude controller.

\subsection{6-DoF dynamic model of RLV}

For the sake of simplicity, it is reasonable to assume that the impact of Earths rotation on the flight control system is not taken into consideration in this work [5], and RLV is regarded as an unpowered rigid body flight vehicle during its reentry phase. The complete application model of RLV is given as depicted in Fig. 1. Generally, the 6-DoF dynamic model of RLV can be separated into the 3-DoF translational kinematic equations, and the 3-DoF rotational kinematic equations, which can be derived based on Ref. [41][42][43].

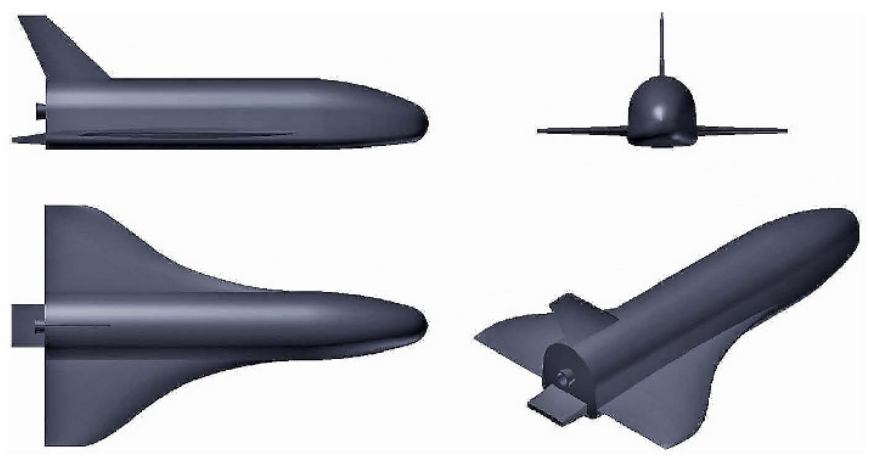

Fig. 1. The complete application model of RLV

The 3-DoF translational kinematic equations are stated by six first-order equations as follows:

$$
\begin{aligned}
\dot{x}= & V \cdot \cos \gamma \cdot \cos \chi \\
\dot{y}= & V \cdot \cos \gamma \cdot \sin \chi \\
\dot{z}= & V \cdot \sin \gamma \\
\dot{V}= & \frac{1}{m}(-D-m g \sin \gamma) \\
\dot{\alpha}= & q-\tan \beta(p \cos \alpha+r \sin \alpha) \\
& +\frac{1}{m V \cos \beta}[-L+m g \cos \gamma \cos \mu] \\
\dot{\beta}= & p \sin \alpha-r \cos \alpha+\frac{1}{m V}[Y+m g \cos \gamma \sin \mu]
\end{aligned}
$$


where $x, y$ and $z$ denote the location of RLV referenced to the flight-path coordinate frame. $V$ represents the flight velocity, and $\gamma, \chi$ and $\mu$ are the flight path angle, heading angle and bank angle, respectively. $\alpha, \beta$ are the angles of attack (AOA) and sideslip angle respectively. $p, q$ and $r$ are the roll, pitch and yaw rates, respectively. $L, D$ and $Y$ are aerodynamic lift, drag and side forces as described in (6).

The 3-DoF rotational kinematic equations are described by six first-order equations as follows:

$\dot{p}=\left(c_{1} r+c_{2} p\right) q+c_{3} \bar{L}+c_{4} N$

$\dot{q}=c_{5} p r-c_{6}\left(p^{2}-r^{2}\right)+c_{7} M$

$\dot{r}=\left(c_{8} p-c_{2} r\right) q+c_{4} \bar{L}+c_{9} N$

$\dot{\phi}=p+(q \sin \phi+r \cos \phi) \tan \theta$

$\dot{\theta}=q \cos \phi-r \sin \phi$

$\dot{\psi}=q \sin \phi \sec \theta+r \cos \phi \sec \theta$

where $\bar{L}, M$ and $N$ are the rolling, pitching and yawing aerodynamic moments described in (7). $c_{1}, \ldots, c_{9}$ are stated in (5). $\phi, \theta$ and $\psi$ are the roll, pitch and yaw angles respectively,

$c_{1}=\frac{\left(J_{y y}-J_{z z}\right) J_{z z}-J_{x z}^{2}}{a}, c_{2}=\frac{\left(J_{x x}-J_{y y}+J_{z z}\right) J_{x z}}{a}$

$c_{3}=\frac{J_{z z}}{a}, c_{4}=\frac{J_{x z}}{a}, c_{5}=\frac{\left(J_{z z}-J_{x x}\right)}{J_{y y}}, c_{6}=\frac{J_{x z}}{J_{y y}}$

$c_{7}=\frac{1}{J_{y y}}, c_{8}=\frac{J_{x x}\left(J_{x x}-J_{y y}\right)+J_{x z}^{2}}{a}, c_{9}=\frac{J_{x x}}{a}$

where $a=J_{x x} J_{z z}-J_{x z}^{2}, J_{x x}, J_{y y}$ and $J_{z z}$ and are the moments of inertia produced by rotating around $x, y, z$ axis. $J_{x y}=J_{y x}=$ $J_{y z}=J_{z y}=0$ are correct for the reason that RLV is symmetric around symmetry plane of $O_{x z} . J_{x z}=J_{z x}$ are inertial product.

$L=\bar{q} S \bar{b} C_{L}, D=\bar{q} S \bar{c} C_{D}, Y=\bar{q} S \bar{b} C_{Y}$

$\bar{L}=\bar{q} S \bar{b} C_{l}, M=\bar{q} S \bar{c} C_{m}, N=\bar{q} S \bar{b} C_{n}$

where $\bar{q}=0.5 \rho V^{2}$ represents dynamic pressure, $\rho$ is the air density. $S$ represents the wing reference area, $\bar{b}$ is the wing span and $\bar{c}$ is the wing mean geometric chord. $C_{L}, C_{D}, C_{Y}$ are the coefficients of lift, drag, side forces, respectively, $C_{l}, C_{m}, C_{n}$ are the rolling, pitching and yawing moment coefficients described as follows

$C_{L}=C_{L}^{0}+C_{L}^{\alpha} \alpha+C_{L}^{\beta} \beta+C_{L}^{\delta_{e}} \delta_{e}$

$C_{D}=C_{D}^{0}+C_{D}^{\alpha} \alpha+C_{D}^{\beta} \beta+C_{D}^{\delta_{e}} \delta_{e}$

$C_{Y}=C_{Y}^{\beta} \beta+C_{Y}^{\delta_{r}} \delta_{r}$

$C_{l}=C_{l}^{\beta} \beta+C_{l}^{\delta_{a}} \delta_{a}+C_{l}^{\delta_{r}} \delta_{r}+C_{l}^{p}\left(\frac{p \bar{b}}{2 V}\right)+C_{l}^{r}\left(\frac{r \bar{b}}{2 V}\right)$

$C_{m}=C_{m}^{0}+C_{m}^{\alpha} \alpha+C_{m}^{\beta} \beta+C_{m}^{\delta_{e}} \delta_{e}+C_{l}^{q}\left(\frac{q \bar{c}}{2 V}\right)$

$C_{n}=C_{n}^{\beta} \beta+C_{n}^{\delta_{a}} \delta_{a}+C_{n}^{\delta_{r}} \delta_{r}+C_{n}^{p}\left(\frac{p \bar{b}}{2 V}\right)+C_{n}^{r}\left(\frac{r \bar{b}}{2 V}\right)$

where $\delta_{a}, \delta_{e}, \delta_{r}$ represent the aileron, elevator and rudder deflections of the RLV, respectively. $C_{*}^{*}$ denotes the corresponding aerodynamic derivatives. All parameters are showed in Table. 1 of the section 4 .

\subsection{Control-oriented attitude model}

In general, the 3-DoF translational kinematic equations referenced to a flight-path coordinate system are used to generate flight trajectory and design the guidance system, whereas the 3-DoF rotational kinematic equations referenced to a vehiclebody-fixed coordinate system are employed to design the attitude controller. This paper aims at designing attitude controller for reentry RLV, and hence a control-oriented attitude model will be established by a model transformation on the basis of rotational kinematic equations (3) - (4) for facilitating the attitude controller design.

Substituting (6) and (7) into (3), then the Eq.(3) can be rewritten as follows,

$\left[\begin{array}{c}\dot{p} \\ \dot{q} \\ \dot{r}\end{array}\right]=\left[\begin{array}{c}f_{p} \\ f_{q} \\ f_{r}\end{array}\right]+\boldsymbol{G}_{u}\left[\begin{array}{c}\delta_{a} \\ \delta_{e} \\ \delta_{r}\end{array}\right]$

where $f_{p}, f_{q}, f_{r}$ and $\boldsymbol{G}_{u}$ are described respectively as,

$f_{p}=\left(c_{1} r+c_{2} p\right) q+c_{3} M_{x}+c_{4} M_{z}$

$f_{q}=c_{5} p r-c_{6}\left(p^{2}-r^{2}\right)+c_{7} M_{y}$

$f_{r}=\left(c_{8} p-c_{2} r\right) q+c_{4} M_{x}+c_{9} M_{z}$

$\boldsymbol{G}_{u}=\bar{q} S\left[\begin{array}{ccc}c_{3} & 0 & c_{4} \\ 0 & c_{7} & 0 \\ c_{4} & 0 & c_{9}\end{array}\right]\left[\begin{array}{ccc}\bar{b} C_{l}^{\delta_{a}} & 0 & \bar{b} C_{l}^{\delta_{r}} \\ 0 & \bar{c} C_{m}^{\delta_{e}} & 0 \\ \bar{b} C_{n}^{\delta_{a}} & 0 & \bar{b} C_{n}^{\delta_{r}}\end{array}\right]$

and $M_{x}, M_{y}, M_{z}$ are stated by

$M_{x}=\bar{q} S \bar{b}\left[C_{l}^{\beta} \beta+C_{l}^{p} t\left(\frac{p \bar{b}}{2 V}\right)+C_{l}^{r}\left(\frac{r \bar{b}}{2 V}\right)\right]$

$M_{y}=\bar{q} S \bar{c}\left[C_{m}^{0}+C_{m}^{\alpha} \alpha+C_{m}^{\beta} \beta+C_{l}^{q}\left(\frac{q \bar{c}}{2 V}\right)\right]$

$M_{z}=\bar{q} S \bar{b}\left[C_{n}^{\beta} \beta+C_{n}^{p}\left(\frac{p \bar{b}}{2 V}\right)+C_{n}^{r}\left(\frac{r \bar{b}}{2 V}\right)\right]$

Taking time derivative of (4), the second-derivative of attitude-angle is derived as below,

$[\ddot{\phi}, \ddot{\theta}, \ddot{\psi}]^{T}=\boldsymbol{L}(\phi, \theta)[\dot{p}, \dot{q}, \dot{r}]^{T}+\boldsymbol{g}(\phi, \theta, \psi)$

and substituting (8) into (12), the equation of motion (12) is converted to a second-order nonlinear dynamic model as,

$$
\begin{aligned}
{[\ddot{\phi}, \ddot{\theta}, \ddot{\psi}]^{T} } & =\boldsymbol{L}(\phi, \theta)\left[f_{p}, f_{q}, f_{r}\right]^{T}+\boldsymbol{g}(\phi, \theta, \psi) \\
& +\boldsymbol{L}(\phi, \theta) \boldsymbol{G}_{u}\left[\delta_{a}, \delta_{e}, \delta_{r}\right]^{T}
\end{aligned}
$$

where $L(\phi, \theta), g(\phi, \theta, \psi)$ are depicted respectively as

$L(\phi, \theta)=\left[\begin{array}{ccc}1 & \sin \phi \tan \theta & \cos \phi \tan \theta \\ 0 & \cos \phi & -\sin \phi \\ 0 & \sin \phi \sec \theta & \cos \phi \sec \theta\end{array}\right]$

$g(\phi, \theta, \psi)=\left[\begin{array}{c}\dot{\theta} \dot{\psi} \sec \theta+\dot{\phi} \dot{\theta} \tan \theta \\ -\dot{\phi} \dot{\psi} \cos \theta \\ \dot{\phi} \dot{\theta} \sec \theta+\dot{\theta} \dot{\psi} \tan \theta\end{array}\right]$ 
However, it is worth noting that the wide range flight enrameters and unknown external disturbances. Thus, Eq.(13) is not available to describe the actual model information ex-215 actly. To characterize the reentry flight process as specifically as possible, the parameter uncertainties and external disturbances be considered. And hence a control-oriented attitude model with more practical information is formulated, which is the basis of the attitude controller design in this work,

$$
\begin{gathered}
{[\ddot{\phi}, \ddot{\theta}, \ddot{\psi}]^{T}=\boldsymbol{L}(\phi, \theta)\left[f_{p}, f_{q}, f_{r}\right]^{T}+\boldsymbol{g}(\phi, \theta, \psi)+\left[\Delta f_{1}, \Delta f_{2}, \Delta f_{3}\right]^{T}} \\
+\boldsymbol{L}(\phi, \theta)\left(\boldsymbol{G}_{u}+\Delta \boldsymbol{G}_{u}\right)\left[\delta_{a}, \delta_{e}, \delta_{r}\right]^{T}+\left[d_{1}, d_{2}, d_{3}\right]^{T}
\end{gathered}
$$

where $d_{i}(i=1,2,3)$ denote external disturbances; $\Delta f_{i}$ and $\Delta \boldsymbol{G}_{u}$ denote parameter uncertainties, which can be stated detailly as

$$
\begin{gathered}
{\left[\Delta f_{1}, \Delta f_{2}, \Delta f_{3}\right]^{T}=\boldsymbol{L}(\phi, \theta)\left[c_{3} \Delta M_{x}+c_{4} \Delta M_{z}, c_{7} \Delta M_{y}, c_{9} \Delta M_{z}\right]^{T}} \\
\Delta \boldsymbol{G}_{u}=\bar{q} S\left[\begin{array}{ccc}
c_{3} & 0 & c_{4} \\
0 & c_{7} & 0 \\
c_{4} & 0 & c_{9}
\end{array}\right]\left[\begin{array}{ccc}
\bar{b} \Delta C_{l}^{\delta_{a}} & 0 & \bar{b} \Delta C_{l}^{\delta_{r}} \\
0 & \bar{c} \Delta C_{m}^{\delta_{e}} & 0 \\
\bar{b} \Delta C_{n}^{\delta_{a}} & 0 & \bar{b} \Delta C_{n}^{\delta_{r}}
\end{array}\right]
\end{gathered}
$$

where $\Delta M_{x}, \Delta M_{y}$ and $\Delta M_{z}$ denote uncertainty terms induced by the perturbations of aerodynamic parameters, which are described respectively as,

$$
\begin{aligned}
& \Delta M_{x}=\bar{q} \cdot S \cdot \bar{b}\left[\Delta C_{l}^{\beta} \cdot \beta+\Delta C_{l}^{p} \cdot\left(\frac{p \bar{b}}{2 V}\right)+\Delta C_{l}^{r} \cdot\left(\frac{r \bar{b}}{2 V}\right)\right] \\
& \Delta M_{y}=\bar{q} \cdot S \cdot \bar{c}\left[\Delta C_{m}^{0}+\Delta C_{m}^{\alpha} \cdot \alpha+\Delta C_{m}^{\beta} \cdot \beta+\Delta C_{m}^{q} \cdot\left(\frac{q \bar{c}}{2 V}\right)\right] \\
& \Delta M_{z}=\bar{q} \cdot S \cdot \bar{b}\left[\Delta C_{n}^{\beta} \cdot \beta+\Delta C_{n}^{p} \cdot\left(\frac{p \bar{b}}{2 V}\right)+\Delta C_{n}^{r} \cdot\left(\frac{r \bar{b}}{2 V}\right)\right]
\end{aligned}
$$

where $\Delta C_{*}^{*}$ represents the corresponding aerodynamic coeffi-240 cient perturbation of RLVs.

For designing the attitude controller succinctly, the attitude control model in (16) can be simplified as a second-order nonlinear system as following,

$$
\begin{aligned}
& \ddot{x}=f(x)+\Delta f(x)+(g(x)+\Delta g(x)) u+d \\
& y=x
\end{aligned}
$$

where $\boldsymbol{x}=\left[x_{1}, x_{2}, x_{3}\right]^{T}=[\varphi, \theta, \psi]^{T}$ is the vehicle state vector, $\boldsymbol{y}=\left[y_{1}, y_{2}, y_{3}\right]^{T}$ is the system output vector, $\boldsymbol{u}=$ 约, $\left.u_{2}, u_{3}\right]^{T}=\left[\delta_{a}, \delta_{e}, \delta_{r}\right]^{T}$ is the control input vector genetated by aerodynamic surface; $\boldsymbol{f}(\boldsymbol{x})=\boldsymbol{L}(\phi, \theta)\left[f_{p}, f_{q}, f_{r}\right]^{T}+\boldsymbol{g}(\phi, \theta, \psi)=$ $\left[f_{1}(\boldsymbol{x}), f_{2}(\boldsymbol{x}), f_{3}(\boldsymbol{x})\right]^{T}$ and $\boldsymbol{g}(\boldsymbol{x})=\boldsymbol{L}(\phi, \theta) \boldsymbol{G}_{u}=\left[g_{i j}(\boldsymbol{x})\right] \in R^{3 \times 3} \mathrm{de}-$ note the system function matrix; $\Delta \boldsymbol{f}(\boldsymbol{x})=\left[\Delta f_{1}, \Delta f_{2}, \Delta f_{3}\right]^{T}$ and $\Delta \boldsymbol{g}(\boldsymbol{x})=\boldsymbol{L}(\phi, \theta) \Delta \boldsymbol{G}_{u}=\left[\Delta \boldsymbol{g}_{i j}(\boldsymbol{x})\right] \in R^{3 \times 3}$ denote parameter un205 certainties; $\boldsymbol{d}=\left[d_{1}, d_{2}, d_{3}\right]^{T}$ represents external disturbance.

The following notation is adopted throughout this paper for the convenience of controller design and stability analysis.

Definition 1: $\Delta \boldsymbol{f}_{s}(\boldsymbol{x})=\Delta \boldsymbol{f}(\boldsymbol{x})+\boldsymbol{d}=\left[\Delta f_{s 1}(\boldsymbol{x}), \Delta f_{s 2}(\boldsymbol{x}), \Delta f_{s 3}(\boldsymbol{x})\right]^{T}$ denotes the uncertainty term of system function matrix $\boldsymbol{f}(\boldsymbol{x})$.

Further, the following assumption is taken into account in the flight controller design.
Assumption 1: The system parameter uncertainties $\Delta f(x)$, $\Delta \boldsymbol{g}(\boldsymbol{x})$ and external disturbance $\boldsymbol{d}$ are bounded, i.e., there exist known bounded positive function matrix $\Delta \boldsymbol{f}_{L}(\boldsymbol{x}), \Delta \boldsymbol{f}_{H}(\boldsymbol{x}) \in$ $R^{3 \times 1}, \Delta \boldsymbol{g}_{L}(\boldsymbol{x}), \Delta \boldsymbol{g}_{H}(\boldsymbol{x}) \in R^{3 \times 3}$ and $\boldsymbol{d}_{H}(\boldsymbol{x}) \in R^{3 \times 1}$, such that for any state vector $\boldsymbol{x}, 0<\Delta f_{i L}(\boldsymbol{x})<\Delta f_{i}(\boldsymbol{x}) \leq \Delta f_{i H}(\boldsymbol{x})$, $0<\Delta g_{i j L}(\boldsymbol{x})<\Delta g_{i j}(\boldsymbol{x}) \leq \Delta g_{i j H}(\boldsymbol{x})$ and $\left|d_{i}(\boldsymbol{x})\right| \leq d_{i H}(\boldsymbol{x})$ hold, where $|\cdot|$ denotes the absolute value, and $i, j=1,2,3$.

\subsection{Control objective}

Assume that the guidance command of attitude angular is $\boldsymbol{y}_{d}=[\phi, \theta, \psi]^{T}$ with a continuous and bounded and derivative $\dot{y}_{d}$. The tracking error of attitude angular is defined as $e_{i}=y_{i d}-y_{i}$ and the attitude tracking error vector of flight attitude is denoted as $\boldsymbol{E}_{i}=\left(e_{i}, \dot{e}_{i}\right)^{\mathrm{T}}(i=1,2,3)$. Noting that the uncertainty terms caused by the aerodynamic parameter perturbations will affect the practical control performance during the reentry phase and then reduce the actual attitude tracking performance of the reusable launch vehicle. Thus, the flight control objective in this paper aims at designing a proper control law $\boldsymbol{u}$ for attitude control system (16) and (20) to guarantee that the actual output $\boldsymbol{y}$ can track the desired command $\boldsymbol{y}_{d}$ well in spite of parameter uncertainties and external disturbances while the close-loop control system of reentry RLV can achieve asymptotic stability simultaneously, i.e.,

$$
\lim _{t \rightarrow \infty}\left\|\phi_{d}-y_{1}\right\|=0, \lim _{t \rightarrow \infty}\left\|\theta_{d}-y_{2}\right\|=0, \lim _{t \rightarrow \infty}\left\|\psi_{d}-y_{3}\right\|=0
$$

The overall control framework of attitude control system in this research is presented in Fig. 2. As depicted in Fig. 2, the control scheme is composed of two controllers designed via CAFHC: the attitude tracking controller utilizing adaptive laws is employed to improve the attitude tracking performance, and the attitude stabilization controller using estimation laws is applied to guarantee the system robustness and the boundness of approximation error. And the detailed design of this control structure is presented in next section.

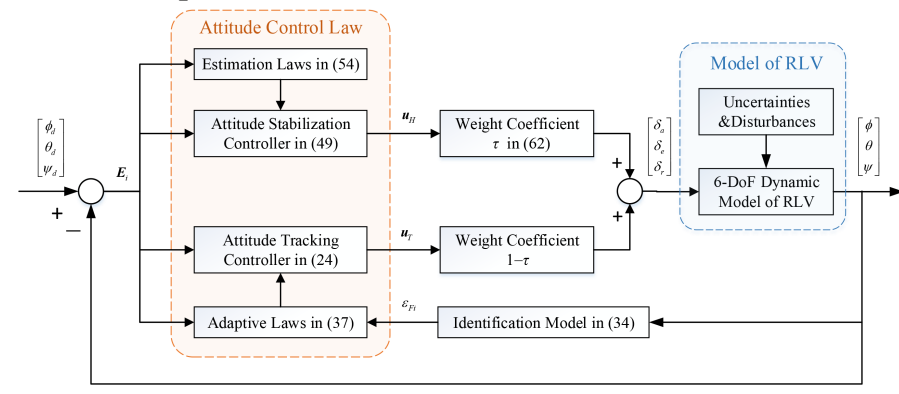

Fig. 2. Framework of attitude control strategy for reentry RLV

\section{Attitude control strategy and stability analysis}

This section focuses on developing attitude controller for reentry RLV to attenuate the adverse effects of various parameter uncertainties and unknown external disturbances and track the expected guidance commands stably and accurately. 


\subsection{Modeling of fuzzy logic system (FLS)}

To develop the attitude controller for RLVs, the following lemma is necessary in this study:

Lemma 1[38]: Suppose the given $f(\boldsymbol{x})$ is a real-continuous function on a compact set $\Omega$, and for any small constant $\varepsilon>^{295}$ 0 , there exists a fuzzy logic system (FLS) $\boldsymbol{\theta}^{T} \boldsymbol{\xi}(\boldsymbol{x})$ such that $\sup _{x \in \Omega}\left|f(\boldsymbol{x})-\theta^{T} \boldsymbol{\xi}(\boldsymbol{x})\right| \leq \varepsilon$ holds.

where $\varepsilon$ is approximation error, $\theta$ denotes a bounded parameter vector, and $\boldsymbol{\xi}(\boldsymbol{x})$ is the fuzzy basis function vector. $\Omega$ denotes the fuzzy approximation region satisfying $\Omega=\left\{x:\|x\| \leq R_{x}\right\}$ and $\bar{\Omega}=R^{3}-\Omega$ is denoted as an area outside the fuzzy approximation region, where $\|\cdot\|$ is Euclidean norm and $R_{x}>0$ is a finite constant.

Although the uncertainty terms $\Delta \boldsymbol{f}_{s}(\boldsymbol{x}), \Delta \boldsymbol{g}(\boldsymbol{x})$ are unknown, ${ }_{300}$ the function vectors of $\Delta f_{s}(x)$ and $\Delta g(x)$ are continuous under the flight conditions considered in this study. Consequently, the namics of $\Delta f_{s}(x)$ and $\Delta g(x)$ can actually be caught by the FLS. Then according to Lemma 1 , for the three-channel attitude angle $x_{i}$ of the RLV, define $N_{i}$ fuzzy sets $F_{i}{ }_{i}{ }\left(l_{i}=1,2, \ldots, N_{i}\right)$ thus the terms $\Delta \boldsymbol{f}_{s}(\boldsymbol{x})$ and $\Delta \boldsymbol{g}(\boldsymbol{x})$ can be approximated by utilizing the FLS respectively, which are detailed as follows,

$\Delta \boldsymbol{f}_{s}(\boldsymbol{x})=\Delta \hat{\boldsymbol{f}}\left(\boldsymbol{x} \mid \boldsymbol{\theta}_{f}\right)+\boldsymbol{\varepsilon}_{f}$

$\Delta \boldsymbol{g}(\boldsymbol{x})=\Delta \hat{\boldsymbol{g}}\left(\boldsymbol{x} \mid \boldsymbol{\theta}_{g}\right)+\boldsymbol{\varepsilon}_{g}$

where $\varepsilon_{f}=\left[\varepsilon_{1 f}, \varepsilon_{2 f}, \varepsilon_{3 f}\right]^{T}$ and $\varepsilon_{g}=\left[\varepsilon_{i j g}\right] \in R^{3 \times 3}$ are vectors of approximation errors, $\boldsymbol{\theta}_{f}=\left[\boldsymbol{\theta}_{1 f}, \boldsymbol{\theta}_{2 f}, \boldsymbol{\theta}_{3 f}\right], \boldsymbol{\theta}_{g}=\left[\boldsymbol{\theta}_{i j g}\right] \in R^{3 \times 3}$ are bounded vectors of corresponding adjusting parameters, and $\boldsymbol{\theta}_{i f} \in R^{1 \times W}, \boldsymbol{\theta}_{i j g} \in R^{1 \times W}(i, j=1,2,3)$, in which $W=\prod_{i=1}^{m} N_{i}$, and $m=3$. $\boldsymbol{\xi}_{f}(x)$ and $\boldsymbol{\xi}_{g}(\boldsymbol{x})$ represent the fuzzy basis function vectors, where $\boldsymbol{\xi}_{(*)}(\boldsymbol{x})=\left[\xi_{1}(\boldsymbol{x}), \ldots, \xi_{W}(\boldsymbol{x})\right]^{T} \in R^{W \times 1}$, and its $l_{1}, \ldots, l_{m}$-th function can be described as follows,

$\xi_{l_{1} \ldots l_{m}(*)}(\boldsymbol{x})=\frac{\prod_{i=1}^{m} \mu_{F_{i} l_{i}}\left(x_{i}\right)}{\sum_{l_{1}=1}^{N_{1}} \cdots \sum_{l_{m}=1}^{N_{m}} \prod_{i=1}^{m} \mu_{F_{i} l_{i}}\left(x_{i}\right)}$

where $\mu_{F_{i} l_{i}}\left(x_{i}\right)$ represents the membership function of $F_{i}^{l_{i}}$ for flight attitude $x_{i}$.

\subsection{Attitude controller design via CAFHC strategy}

To avoid the problem of "approximation capability weakening" for FLS caused by the introduction of H-infinity control term, the attitude control scheme is developed in two steps based on the control-oriented attitude model (16) in this subsection.

\subsubsection{Attitude tracking controller design}

Firstly, the tracking problem of flight attitude for reentry RLV is considered when the attitude angle is in the interior of fuzzy approximation region, i.e. $\boldsymbol{x} \in \Omega$.

Generally, select a suitable vector $\boldsymbol{K}_{i}=\left[k_{i 2}, k_{i 1}\right]$ such that all the roots of polynomial $p(s)=s^{2}+k_{i 1} s+k_{i 2}$ are in the left halfplane of complex plane. Then, the attitude tracking controller can be designed as follows,
$\boldsymbol{u}_{T}=\left[\boldsymbol{g}(\boldsymbol{x})+\Delta \hat{\boldsymbol{g}}\left(\boldsymbol{x} \mid \boldsymbol{\theta}_{g}\right)\right]^{-1}\left[-\boldsymbol{f}(\boldsymbol{x})-\Delta \hat{\boldsymbol{f}}\left(\boldsymbol{x} \mid \boldsymbol{\theta}_{f}\right)+\ddot{\boldsymbol{y}}_{d}+\boldsymbol{K} \boldsymbol{E}\right]+\boldsymbol{u}_{h}{ }^{*}$

where $\boldsymbol{K} \boldsymbol{E}=\left[\boldsymbol{K}_{1} \boldsymbol{E}_{1}, \boldsymbol{K}_{2} \boldsymbol{E}_{2}, \boldsymbol{K}_{3} \boldsymbol{E}_{3}\right]^{T} . \boldsymbol{u}_{h}{ }^{*}$ denotes the H-infinity controller, which is expressed as follows.

$\boldsymbol{u}_{h}^{*}=\left[\boldsymbol{g}(\boldsymbol{x})+\Delta \hat{\boldsymbol{g}}\left(\boldsymbol{x} \mid \boldsymbol{\theta}_{g}\right)\right]^{-1} \boldsymbol{u}_{h}$

where $\boldsymbol{u}_{h}=\left[\boldsymbol{E}_{1}^{T} \boldsymbol{P}_{1} \boldsymbol{B}_{1} / r_{1}, \boldsymbol{E}_{2}^{T} \boldsymbol{P}_{2} \boldsymbol{B}_{2} / r_{2}, \boldsymbol{E}_{3}^{T} \boldsymbol{P}_{3} \boldsymbol{B}_{3} / r_{3}\right]^{T}$. Parameter $r_{i}>0$, and the matix $\boldsymbol{P}_{i}=\boldsymbol{P}_{i}^{T}>0$ satisfy the following Riccati-equation,

$\boldsymbol{P}_{i} \boldsymbol{A}_{i}+\boldsymbol{A}_{i}^{T} \boldsymbol{P}_{i}+\boldsymbol{Q}_{i}+\boldsymbol{P}_{i} \boldsymbol{B}_{i}\left(\frac{1}{\rho_{i}^{2}}-\frac{2}{r_{i}}\right) \boldsymbol{B}_{i}^{T} \boldsymbol{P}_{i}=0$.

where $\boldsymbol{A}_{i}=\left[\begin{array}{lll}0 & 1 ;-k_{i 2}{ }^{i}-k_{i 1}\end{array}\right], \boldsymbol{B}_{i}=[0,1]^{T} . \boldsymbol{Q}_{i}$ denotes a positive-definite symmetric matrix.

According to the Eqs.(20) and (24), the two dynamic equations are obtained respectively as follows,

$\ddot{y}=\boldsymbol{f}(\boldsymbol{x})+\Delta \boldsymbol{f}_{s}(\boldsymbol{x})+[\boldsymbol{g}(\boldsymbol{x})+\Delta \boldsymbol{g}(\boldsymbol{x})] \boldsymbol{u}_{T}$

$\ddot{\boldsymbol{y}}_{d}=-\boldsymbol{K} \boldsymbol{E}+\boldsymbol{f}(\boldsymbol{x})+\Delta \hat{\boldsymbol{f}}\left(\boldsymbol{x} \mid \boldsymbol{\theta}_{f}\right)+\left[\boldsymbol{g}(\boldsymbol{x})+\Delta \hat{\boldsymbol{g}}\left(\boldsymbol{x} \mid \boldsymbol{\theta}_{g}\right)\right] \boldsymbol{u}_{T}-\boldsymbol{u}_{h}$

Subtracting (27) from (28) and carrying out some mathematical transformation, we obtain the dynamic equation of attitude tracking error vector for reentry RLV as,

$$
\begin{gathered}
\dot{\boldsymbol{E}}_{i}=\boldsymbol{A}_{i} \boldsymbol{E}_{i}+\boldsymbol{B}_{i}\left\{\Delta \hat{f}_{i}\left(\boldsymbol{x} \mid \boldsymbol{\theta}_{i f}\right)-\Delta f_{s i}(\boldsymbol{x})+\right. \\
\left.\sum_{j=1}^{3}\left[\Delta \hat{g}_{i j}\left(\boldsymbol{x} \mid \boldsymbol{\theta}_{i j g}\right)-\Delta g_{i j}(\boldsymbol{x})\right] u_{T j}-u_{h i}\right\}
\end{gathered}
$$

Definition 2: Based on the above Eq.(29), the fuzzy modeling error $w_{i}$ and optimal fuzzy modeling error $w_{i}^{*}$ of the RLV attitude control system in this paper are defined as follows,

$w_{i}=\Delta f_{s i}(\boldsymbol{x})-\Delta \hat{f}_{i}\left(\boldsymbol{x} \mid \boldsymbol{\theta}_{i f}\right)+\sum_{j=1}^{3}\left[\Delta g_{i j}(\boldsymbol{x})-\Delta \hat{g}_{i j}\left(\boldsymbol{x} \mid \boldsymbol{\theta}_{i j g}\right)\right] u_{T j}$
$w_{i}^{*}=\Delta f_{s i}(\boldsymbol{x})-\Delta \hat{f}_{i}\left(\boldsymbol{x} \mid \boldsymbol{\theta}_{i f}^{*}\right)+\sum_{j=1}^{3}\left[\Delta g_{i j}(\boldsymbol{x})-\Delta \hat{g}_{i j}\left(\boldsymbol{x} \mid \boldsymbol{\theta}_{i j g}^{*}\right)\right] u_{T j}$

where $\boldsymbol{\theta}_{i f}^{*}, \boldsymbol{\theta}_{i j g}^{*}$ denote the vectors of optimal parameters, which are stated respectively as,

$$
\begin{aligned}
& \boldsymbol{\theta}_{i f}^{*}=\arg \min _{\boldsymbol{\theta}_{i f} \in \Omega_{f}}\left[\sup _{\boldsymbol{x} \in \Omega}\left|\Delta f_{s i}(\boldsymbol{x})-\Delta \hat{f}_{i}\left(\boldsymbol{x} \mid \boldsymbol{\theta}_{i f}\right)\right|\right] \\
& \boldsymbol{\theta}_{i j g}^{*}=\arg \min _{\boldsymbol{\theta}_{i j g} \in \Omega_{g}}\left[\sup _{\boldsymbol{x} \in \Omega}\left|\Delta g_{i j}(\boldsymbol{x})-\Delta \hat{g}_{i j}\left(\boldsymbol{x} \mid \boldsymbol{\theta}_{i j g}\right)\right|\right]
\end{aligned}
$$

where $\Omega_{f}$ and $\Omega_{g}$ represent the bounded compact sets of the adjusting parameter vectors $\boldsymbol{\theta}_{i f}$ and $\boldsymbol{\theta}_{i j g}$ respectively.

Assume $\tilde{\boldsymbol{\theta}}_{i f}=\boldsymbol{\theta}_{i f}-\boldsymbol{\theta}_{i f}^{*}, \tilde{\boldsymbol{\theta}}_{i j g}=\boldsymbol{\theta}_{i j g}-\boldsymbol{\theta}_{i j g}^{*}$. Then the dynamic of attitude tracking error vector can be further described as,

$\dot{\boldsymbol{E}}_{i}=\boldsymbol{A}_{i} \boldsymbol{E}_{i}+\boldsymbol{B}_{i}\left[\tilde{\boldsymbol{\theta}}_{i f}^{T} \boldsymbol{\xi}_{f}(\boldsymbol{x})+\sum_{j=1}^{3} \tilde{\boldsymbol{\theta}}_{i j g}^{T} \boldsymbol{\xi}_{g}(\boldsymbol{x}) u_{T j}-u_{h i}-w_{i}^{*}\right]$

In order to obtain the adaptive laws of system parameters, the filter modeling error of attitude angular is denoted as $\varepsilon_{F i}=$ $\dot{\hat{x}}_{i}-\dot{x}_{i}$, and the identification model combined with a low-pass filter [44] is employed as follows,

$$
\begin{gathered}
\ddot{\hat{x}}_{i}=-\alpha_{F i} \varepsilon_{F i}+f_{i}(\boldsymbol{x})+\Delta \hat{f}_{i}\left(\boldsymbol{x} \mid \boldsymbol{\theta}_{i f}\right)+ \\
\sum_{j=1}^{3}\left[g_{i j}(\boldsymbol{x})+\Delta \hat{g}_{i j}\left(\boldsymbol{x} \mid \boldsymbol{\theta}_{i j g}\right)\right] u_{T j}+v_{i}
\end{gathered}
$$


where $\hat{x}_{i}$ is the estimated value of attitude angle $x_{i}, \alpha_{F i}>0$ the filter parameter pre-set by the designer. And $v_{i}$ denotes the compensation term of identification model, which can be stated by,

$v_{i}=-\beta_{i} \operatorname{sgn}\left(\varepsilon_{F i}\right)$

where $\beta_{i} \geq \bar{w}_{i}$ denotes a finite constant pre-set by the designer, and $\bar{w}_{i}=\sup _{\forall x \in \Omega}\left|w_{i}^{*}\right|$.

Noting that $\dot{\varepsilon}_{F i}=\ddot{\hat{x}}_{i}-\ddot{x}_{i}$ according to the expression of the filtering modeling error. And subtracting Eq.(27) from Eq.(34), then the dynamic of filtering modeling error can be derived as,

$\dot{\varepsilon}_{F i}=-\alpha_{F i} \varepsilon_{F i}-w_{i}^{*}+\tilde{\boldsymbol{\theta}}_{i f}^{T} \boldsymbol{\xi}_{f}(\boldsymbol{x})+v_{i}+\sum_{j=1}^{3} \tilde{\boldsymbol{\theta}}_{i j g}^{T} \boldsymbol{\xi}_{g}(\boldsymbol{x}) u_{T j}$

Accordingly, the adaptive laws of parameter vectors $\boldsymbol{\theta}_{i f}$ and $\boldsymbol{\theta}_{i j g}$ are developed on the basis of the Eq.(33) and above dynamic equation (36) respectively as below,

$$
\begin{aligned}
& \dot{\boldsymbol{\theta}}_{i f}=-\gamma_{i f}\left(\boldsymbol{E}_{i}^{T} \boldsymbol{P}_{i} \boldsymbol{B}_{i}+\gamma_{i \varepsilon} \varepsilon_{F i}\right) \boldsymbol{\xi}_{f}(\boldsymbol{x}), \\
& \dot{\boldsymbol{\theta}}_{i j g}=-\gamma_{i j g}\left(\boldsymbol{E}_{i}^{T} \boldsymbol{P}_{i} \boldsymbol{B}_{i}+\gamma_{i \varepsilon} \varepsilon_{F i}\right) \boldsymbol{\xi}_{g}(\boldsymbol{x}) u_{T i} .
\end{aligned}
$$

where $\gamma_{i f}, \gamma_{i j g}, \gamma_{i \varepsilon} \in R^{+}$denote the learning rates.

Theorem 1: Considering the attitude control system (16) and (20) for reentry RLV, given that the Assumption 1 and Lemma 1 are satisfied when the aircraft attitude $x \in \Omega$. The attitude track-355 ing controller is chosen as (24) together with the identification model (34) and the adaptive laws are designed as (37), then the closed-loop control system can achieve semi-global asymptotic stable, and the attitude tracking error vector $\boldsymbol{E}_{i}$ and filter modeling error vector $\varepsilon_{F i}$ can both converge to a small neighborhood $D_{T E}, D_{\varepsilon}$ around origin through tuning the designed controller parameters appropriately.

$$
\begin{aligned}
& D_{T E}=\left\{\boldsymbol{E}_{i}:\left\|\boldsymbol{E}_{i}\right\| \leq \rho_{i} \bar{w}_{i}\left(\lambda_{\min }\left(\boldsymbol{Q}_{i}\right)\right)^{-1 / 2}\right\}, \\
& D_{\varepsilon}=\left\{\varepsilon_{F i}:\left|\varepsilon_{F i}\right| \leq \rho_{i} \bar{w}_{i}\left(\gamma_{i \varepsilon} \alpha_{F i}\right)^{-1 / 2}\right\}
\end{aligned}
$$

where $\lambda_{\min }\left(\boldsymbol{Q}_{i}\right)$ is the minimum eigenvalue of matrix $\boldsymbol{Q}_{i}$.

\section{Proof:}

Choose the Lyapunov candidate function $V_{T i}$ about the flight ${ }_{360}$ attitude dynamic as

$V_{T i}=\frac{1}{2} \boldsymbol{E}_{i}^{T} \boldsymbol{P}_{i} \boldsymbol{E}_{i}+\frac{1}{2} \gamma_{i \varepsilon} \varepsilon_{F i}^{2}+\frac{1}{2 \gamma_{i f}} \tilde{\boldsymbol{\theta}}_{i f}^{T} \tilde{\boldsymbol{\theta}}_{i f}+\sum_{j=1}^{3} \frac{1}{2 \gamma_{i j g}} \tilde{\boldsymbol{\theta}}_{i j g}^{T} \tilde{\boldsymbol{\theta}}_{i j g}$

Taking the derivative of $V_{T i}$ along Eqs.(24) and (33) yields,

$$
\begin{aligned}
\dot{V}_{T i}= & -\frac{1}{2} \boldsymbol{E}_{i}^{T} \boldsymbol{Q}_{i} \boldsymbol{E}_{i}+\gamma_{i \varepsilon} \boldsymbol{\varepsilon}_{F i} \tilde{\boldsymbol{\theta}}_{i f}^{T} \boldsymbol{\xi}_{f}(\boldsymbol{x})+\frac{1}{\gamma_{i f}} \tilde{\boldsymbol{\theta}}_{i f}^{T} \dot{\tilde{\boldsymbol{\theta}}}_{i f}-\gamma_{i \varepsilon} \alpha_{F i} \varepsilon_{F i}^{2} \\
& +\boldsymbol{E}_{i}{ }^{T} \boldsymbol{P}_{i} \boldsymbol{B}_{i}\left[\tilde{\boldsymbol{\theta}}_{i f}^{T} \boldsymbol{\xi}_{f}(\boldsymbol{x})+\sum_{j=1}^{3} \tilde{\boldsymbol{\theta}}_{i j g}^{T} \boldsymbol{\xi}_{g}(\boldsymbol{x}) u_{T j}-u_{h i}-w_{i}^{*}\right]+ \\
& \frac{1}{\gamma_{i j g}} \sum_{j=1}^{3} \tilde{\boldsymbol{\theta}}_{i j g}^{T} \dot{\tilde{\boldsymbol{\theta}}}_{i j g}+\gamma_{i \varepsilon} \boldsymbol{\varepsilon}_{F i} \sum_{j=1}^{3} \tilde{\boldsymbol{\theta}}_{i j g}^{T} \boldsymbol{\xi}_{g}(\boldsymbol{x}) u_{T j}+\gamma_{i \varepsilon} \boldsymbol{\varepsilon}_{F i}\left(v_{i}-w_{i}^{*}\right)
\end{aligned}
$$

Substituting (25), (35) and the Riccati-equation (26) into (40), $\dot{V}_{T i}$ can be rewritten as,

$$
\begin{aligned}
\dot{V}_{T i}= & -\frac{1}{2} \boldsymbol{E}_{i}{ }^{T} \boldsymbol{Q}_{i} \boldsymbol{E}_{i}-\gamma_{i \varepsilon} \alpha_{F i} \varepsilon_{F i}^{2}+\frac{1}{2 \rho_{i}^{2}}\left(\boldsymbol{E}_{i}^{T} \boldsymbol{P}_{i} \boldsymbol{B}_{i}\right)^{2}+\tilde{\boldsymbol{\theta}}_{i f}^{T}\left[\left(\boldsymbol{E}_{i}^{T} \boldsymbol{P}_{i} \boldsymbol{B}_{i}\right.\right. \\
& \left.\left.+\gamma_{i \varepsilon} \varepsilon_{F i}\right) \boldsymbol{\xi}_{f}(\boldsymbol{x})+\frac{1}{\gamma_{i f}} \dot{\boldsymbol{\theta}}_{i f}\right]-\boldsymbol{E}_{i}{ }^{T} \boldsymbol{P}_{i} \boldsymbol{B}_{i} w_{i}^{*}+\sum_{j=1}^{3} \tilde{\boldsymbol{\theta}}_{i j g}^{T}\left[\left(\boldsymbol{E}_{i}{ }^{T} \boldsymbol{P}_{i} \boldsymbol{B}_{i}\right.\right. \\
& \left.\left.+\gamma_{i \varepsilon} \varepsilon_{F i}\right) \boldsymbol{\xi}_{g}(\boldsymbol{x}) u_{T j}+\sum_{j=1}^{3} \frac{1}{\gamma_{i j g}} \dot{\boldsymbol{\theta}}_{i j g}\right]
\end{aligned}
$$

The adaptive laws of parameter vectors (37) are applied to the above formulation (41), which can be transformed into,

$$
\begin{aligned}
\dot{V}_{T i}= & -\frac{1}{2} \boldsymbol{E}_{i}^{T} \boldsymbol{Q}_{i} \boldsymbol{E}_{i}+\frac{1}{2 \rho_{i}^{2}}\left(\boldsymbol{E}_{i}^{T} \boldsymbol{P}_{i} \boldsymbol{B}_{i}\right)^{2}-\gamma_{i \varepsilon} \alpha_{F i} \varepsilon_{F i}^{2}-\boldsymbol{E}_{i}^{T} \boldsymbol{P}_{i} \boldsymbol{B}_{i} w_{i}^{*} \\
& =\frac{1}{2}\left[\left(\rho_{i} w_{i}^{*}\right)^{2}-\boldsymbol{E}_{i}^{T} \boldsymbol{Q}_{i} \boldsymbol{E}_{i}-\left(\frac{\boldsymbol{E}_{i}^{T} \boldsymbol{P}_{i} \boldsymbol{B}_{i}}{\rho_{i}}+\rho_{i} w_{i}^{*}\right)^{2}\right]-\gamma_{i \varepsilon} \alpha_{F i} \varepsilon_{F i}^{2} \\
& \leq-\frac{1}{2} \boldsymbol{E}_{i}^{T} \boldsymbol{Q}_{i} \boldsymbol{E}_{i}+\frac{1}{2}\left(\rho_{i} w_{i}^{*}\right)^{2}-\gamma_{i \varepsilon} \alpha_{F i} \varepsilon_{F i}^{2}
\end{aligned}
$$

As $\boldsymbol{Q}_{i}$ is a positive-definite symmetric matrix, it can be restated as $\boldsymbol{Q}_{i}=\boldsymbol{U}_{i}{ }^{T} \Lambda_{i} \boldsymbol{U}_{i}$ based on Ref.[45], where $\boldsymbol{U}_{i}$ denotes a unitary-matrix fulfilling $\boldsymbol{U}_{i}^{T} \boldsymbol{U}_{i}=\boldsymbol{I}$, and $\Lambda_{i}$ is a diagonal-matrix including all eigenvalues of $\boldsymbol{Q}_{i}$. Consequently, the following inequity is formulated,

$\lambda_{\min }\left(\boldsymbol{Q}_{i}\right)\left\|\boldsymbol{E}_{i}\right\|^{2} \leq \boldsymbol{E}_{i}^{T} \boldsymbol{Q}_{i} \boldsymbol{E}_{i}$

Using the above inequality, then expression in (42) becomes,

$$
\begin{aligned}
\dot{V}_{T i} & \leq \frac{1}{2} \lambda_{\min }\left(\boldsymbol{Q}_{i}\right)\left\|\boldsymbol{E}_{i}\right\|^{2}+\frac{1}{2}\left(\rho_{i} w_{i}^{*}\right)^{2}-\gamma_{i \varepsilon} \alpha_{F i} \varepsilon_{F i}^{2} \\
& \leq \frac{1}{2} \lambda_{\min }\left(\boldsymbol{Q}_{i}\right)\left\|\boldsymbol{E}_{i}\right\|^{2}+\frac{1}{2}\left(\rho_{i} \bar{w}_{i}\right)^{2}-\gamma_{i \varepsilon} \alpha_{F i} \varepsilon_{F i}^{2}
\end{aligned}
$$

and it is easy to prove that

$\dot{V}_{T i} \leq-\frac{1}{2} \lambda_{\min }\left(\boldsymbol{Q}_{i}\right)\left\|\boldsymbol{E}_{i}\right\|^{2}+\frac{1}{2}\left(\rho_{i} \bar{w}_{i}\right)^{2}$

According to the inequality (44), $\dot{V}_{T i}<0$ holds for $\left\|\boldsymbol{E}_{i}\right\|>$ $\rho_{i} \bar{w}_{i}\left(\lambda_{\min }\left(\boldsymbol{Q}_{i}\right)\right)^{-1 / 2}$. Thence the tracking error vetor of attitude angle $\boldsymbol{E}_{i}$ converges to an arbitrarily small neighborhood around origin $D_{T E}$ presented in (38) through tuning the designed parameters and selecting the matrix $\boldsymbol{Q}_{i}$ properly. Similarly, it is easy to demonstrate that $\varepsilon_{F i}$ can converge to the region $D_{\varepsilon}$ given in (38) on the basis of the formulation (44).

Assuming $\tilde{\boldsymbol{E}}_{i}=\left[\boldsymbol{E}_{i}{ }^{T}, \boldsymbol{\varepsilon}_{F i}\right]^{T}, \boldsymbol{Q}_{\boldsymbol{E}_{i}}=\operatorname{diag}\left(\boldsymbol{Q}_{i}, \gamma_{i \varepsilon} \alpha_{F i} / 2\right)$, thus inequality (44) can be further transformed as,

$\dot{V}_{T i} \leq-\frac{1}{2} \tilde{\boldsymbol{E}}_{i}^{T} \boldsymbol{Q}_{\boldsymbol{E}_{i}} \tilde{\boldsymbol{E}}_{i}+\frac{1}{2}\left(\rho_{i} \bar{w}_{i}\right)^{2}$

Inspired by the similar proof in Ref.[46], then the above inequality is derived as follows,

$\dot{V}_{T i} \leq-\kappa_{\min } V_{T i}+\kappa_{\min } V_{c i}$

in which $\kappa_{\min }=\lambda_{\min }\left(\boldsymbol{Q}_{\boldsymbol{E}_{i}}\right) / \lambda_{\min }\left(\boldsymbol{P}_{i}\right), V_{c i} \in R^{+}$represents a finite constant. Then solving (47), we have

$V_{T i}(t) \leq\left(V_{T i}(0)-V_{c i}\right) e^{-\kappa_{\min } t}+V_{c i}$

then $V_{T i}(t) \leq \max \left(V_{T i}(0), V_{c i}\right)$ can be obtained according to (48). Consequently, $V_{T i} \in L_{\infty}$ and it indicates that the states 
$\boldsymbol{E}_{i}, \gamma_{i \varepsilon}, \boldsymbol{\varepsilon}_{F i}, \boldsymbol{\theta}_{i f}, \boldsymbol{\theta}_{i j g} \in L_{\infty}$ hold from Eq.(39). As seen from Eq.(24), $\boldsymbol{u}_{T} \in L_{\infty}$ is obviously correct. Therefore, it can be is semi-global asymptotic stable, and all related signals of this system are uniformly ultimately bounded.

Then the proof completes.

\subsubsection{Attitude stabilization controller design}

The designed attitude tracking controller can guarantee that the closed-loop control system is semi-global stable and all related signals are uniformly ultimately bounded, while make the tracking error vector of attitude angle arbitrarily small. However, the fuzzy control law described in Lemma 1 and the Hinfinity controller given in Eq.(25) hold merely for $\boldsymbol{x} \in \Omega,{ }^{420}$ thus the stabilization problem of flight attitude for reentry RLV should be discussed when the attitude angle is in the exterior of fuzzy approximation region, i.e., $x \in \bar{\Omega}$ and an attitude stabilization controller is designed in this part.

Here, we consider $\boldsymbol{\xi}_{f}(\boldsymbol{x})=\mathbf{0}, \boldsymbol{\xi}_{g}(\boldsymbol{x})=\mathbf{0}$ and $\boldsymbol{u}_{h}{ }^{*}=\mathbf{0}$ when ${ }^{425}$ $x \in \bar{\Omega}$. And the following assumption is provided for later use.

Assumption 2[46]: For any state vector $x \in R^{3}$, the system uncertainty terms $\Delta \boldsymbol{f}_{s}(\boldsymbol{x})$ and $\Delta \boldsymbol{g}(\boldsymbol{x})$ of reentry $R L V$ satisfy the following inequalities,

$-f_{i}^{V}\left(\boldsymbol{\theta}_{i f}^{V} \mid \boldsymbol{x}\right) \leq \Delta f_{s i}(\boldsymbol{x}) \leq f_{i}^{V}\left(\boldsymbol{\theta}_{i f}^{V} \mid \boldsymbol{x}\right)$,

$-g_{i j}(\boldsymbol{x})<-g_{i j}^{V}\left(\boldsymbol{\theta}_{i j g}^{V} \mid \boldsymbol{x}\right) \leq \Delta g_{i j}(\boldsymbol{x}) \leq g_{i j}^{V}\left(\boldsymbol{\theta}_{i j g}^{V} \mid \boldsymbol{x}\right)$

where $f_{i}^{V}\left(\boldsymbol{\theta}_{i f}^{V} \mid \boldsymbol{x}\right)=m_{i f}, g_{i j}^{V}\left(\boldsymbol{\theta}_{i j g}^{V} \mid \boldsymbol{x}\right)=m_{i j g} . \boldsymbol{m}_{f} \in R^{3 \times 1}$ and $\boldsymbol{m}_{g} \in R^{3 \times 3}$ denote the vectors of boundary parameters.

Since the fuzzy logic systems in (21) and (22) and H-infinity controller term (25) are invalid when $x \in \bar{\Omega}$, thus the robust stabilization terms are introduced to design the attitude controller. Then the attitude stabilization controller is designed as

$u_{H}=\left[g(x)+v_{g}\right]^{-1}\left[-f(x)-v_{f}+\ddot{y}_{d}+K E\right]$

where $\boldsymbol{v}_{f}=\left[v_{1 f}, v_{2 f}, v_{3 f}\right]^{T}, \boldsymbol{v}_{g}=\left[v_{i j g}\right] \in R^{3 \times 3}$ represent the robust stabilization terms. And $v_{i f}=-\operatorname{sgn}\left(\boldsymbol{E}_{i}{ }^{T} \boldsymbol{P}_{i} \boldsymbol{B}_{i}\right) \hat{m}_{i f}, v_{i j g}=$ $-\operatorname{sgn}\left(\boldsymbol{E}_{i}{ }^{T} P_{i} B_{i} u_{a i}\right) \hat{m}_{i j g}$, in which $\boldsymbol{u}_{a}=-\boldsymbol{f}(\boldsymbol{x})-\boldsymbol{v}_{f}+\ddot{\boldsymbol{y}}_{d}+\boldsymbol{K} \boldsymbol{E}$, $\boldsymbol{u}_{a}=\left[u_{a 1}, u_{a 2}, u_{a 3}\right]^{T}$, and $\hat{m}_{i f}, \hat{m}_{i j g}$ are the estimated values of $m_{i f}, m_{i j g}$ respectively.

According to the Eqs.(20) and (49), yields the two dynamic equations respectively as follows,

$\ddot{y}=\boldsymbol{f}(\boldsymbol{x})+\Delta \boldsymbol{f}_{s}(\boldsymbol{x})+[\boldsymbol{g}(\boldsymbol{x})+\Delta \boldsymbol{g}(\boldsymbol{x})] \boldsymbol{u}_{T}$

$\ddot{y}_{d}=\boldsymbol{f}(\boldsymbol{x})+\boldsymbol{v}_{f}+\left(\boldsymbol{g}(\boldsymbol{x})+\boldsymbol{v}_{g}\right) \boldsymbol{u}_{H}-\boldsymbol{K} \boldsymbol{E}$

Similarly, subtracting (50) from (51) and carrying out some transformation, the dynamic of attitude tracking error vector can be obtained as,

$\dot{\boldsymbol{E}}_{i}=\boldsymbol{A}_{i} \boldsymbol{E}_{i}+\boldsymbol{B}_{i}\left\{v_{i f}-\Delta f_{s i}(\boldsymbol{x})+\sum_{j=1}^{3}\left[v_{i j g}-\Delta \mathrm{g}_{i j g}(\boldsymbol{x})\right] u_{H j}\right\}$

Due to the proper selection of the parameter vector $\boldsymbol{K}_{i}$ given must exist a unique positive-definite matrix $\boldsymbol{P}_{i}=\boldsymbol{P}_{i}^{T}$ satisfying the following Lyapunov-equation,

$\boldsymbol{A}_{i}^{T} \boldsymbol{P}_{i}+\boldsymbol{P}_{i} \boldsymbol{A}_{i}=-\boldsymbol{Q}_{i}$

where $\boldsymbol{Q}_{i}$ represents an arbitrarily given positive-definite symmetric matrix.

For the purpose of averting the great control effect, the boundary adaptive technique is employed to stabilize the attitude control system (16) of reentry RLV and the estimation laws of boundary parameter vectors are constructed as follows,

$$
\begin{aligned}
& \dot{\hat{m}}_{i f}=\eta_{i f}\left(\left|\boldsymbol{E}_{i}{ }^{T} \boldsymbol{P}_{i} \boldsymbol{B}_{i}\right|-\varepsilon_{i f} \hat{m}_{i f}\right) \\
& \dot{\hat{m}}_{i j g}=\eta_{i j g}\left(\left|\boldsymbol{E}_{i}{ }^{T} \boldsymbol{P}_{i} \boldsymbol{B}_{i} u_{H i}\right|-\varepsilon_{i j g} \hat{m}_{i j g}\right)
\end{aligned}
$$

where $\varepsilon_{i f}, \varepsilon_{i j g}>0$ are small constans pre-set by the designer, $\eta_{i f}, \eta_{i j g}>0$ denote the learning rates

Theorem 2: For the attitude control system (16) and (20) of reentry RLV, suppose that the Assumptions 1-2 are fulfilled when the aircraft attitude $x \in \bar{\Omega}$. The attitude stabilization controller is designed as (49) together with the estimation laws of boundary parameter vectors selected as (54), then the closedloop control system can achieve semi-global asymptotic stable, and the attitude tracking error vector $\boldsymbol{E}_{i}$ converges to a small neighborhood $D_{H E}$ around origin by tuning the controller parameters appropriately.

$$
D_{H E}=\left\{\boldsymbol{E}_{i}:\left\|\boldsymbol{E}_{i}\right\| \leq\left\{\left[2 \lambda_{\min }\left(\boldsymbol{Q}_{i}\right)\right]^{-1}\left(\varepsilon_{i f} m_{i f}^{2}+\sum_{j=1}^{3} \varepsilon_{i j g} m_{i j g}^{2}\right)\right\}^{\frac{1}{2}}\right\}
$$

Proof:

Consider the Lyapunov candidate function $V_{H i}$ about the flight attitude dynamic as

$V_{H i}=\frac{1}{2} \boldsymbol{E}_{i}^{T} \boldsymbol{P}_{i} \boldsymbol{E}_{i}+\frac{1}{2 \eta_{i f}} \tilde{m}_{i f}^{2}+\sum_{j=1}^{3} \frac{1}{2 \eta_{i j g}} \tilde{m}_{i j g}^{2}$

where $\tilde{m}_{i f}=\hat{m}_{i f}-m_{i f}, \tilde{m}_{i j g}=\hat{m}_{i j g}-m_{i j g}$.

Differentiating (56) with repect to time along the attitude traking error dynamic (52) and utilizing (53), then

$$
\begin{aligned}
\dot{V}_{H i} & =-\frac{1}{2} \boldsymbol{E}_{i}{ }^{T} \boldsymbol{Q}_{i} \boldsymbol{E}_{i}+\frac{1}{\eta_{i f}} \tilde{m}_{i f} \dot{\hat{m}}_{i f}+\sum_{j=1}^{3} \frac{1}{\eta_{i j g}} \tilde{m}_{i j g} \dot{\hat{m}}_{i j g}+\boldsymbol{E}_{i}{ }^{T} \boldsymbol{P}_{i} \boldsymbol{B}_{i}\left\{v_{i f}\right. \\
& \left.-\Delta f_{s i}(\boldsymbol{x})+\sum_{j=1}^{3}\left[v_{i j g}-\Delta \mathrm{g}_{i j}(\boldsymbol{x})\right] u_{H j}\right\}
\end{aligned}
$$

Substituting the estimation laws (54) into (57), $\dot{V}_{H i}$ becomes,

$$
\begin{aligned}
\dot{V}_{H i}= & -\frac{1}{2} \boldsymbol{E}_{i}^{T} \boldsymbol{Q}_{i} \boldsymbol{E}_{i}-E_{i}^{T} P_{i} B_{i}\left\{\operatorname{sgn}\left(\boldsymbol{E}_{i}^{T} \boldsymbol{P}_{i} \boldsymbol{B}_{i}\right) \hat{m}_{i f}+\Delta f_{s i}(\boldsymbol{x})+\right. \\
& \left.\sum_{j=1}^{3}\left[\operatorname{sgn}\left(\boldsymbol{E}_{i}{ }^{T} \boldsymbol{P}_{i} \boldsymbol{B}_{i} u_{a i}\right) \hat{m}_{i j g}+\Delta g_{i j g}(\boldsymbol{x})\right] u_{H j}\right\}+\tilde{m}_{i f}\left(\left|\boldsymbol{E}_{i}{ }^{T} \boldsymbol{P}_{i} \boldsymbol{B}_{i}\right|\right. \\
& \left.-\varepsilon_{i f} \hat{m}_{i f}\right)+\sum_{j=1}^{3} \tilde{m}_{i j g}\left[\left|\boldsymbol{E}_{i}{ }^{T} \boldsymbol{P}_{i} \boldsymbol{B}_{i} u_{H i}\right|-\varepsilon_{i j g} \hat{m}_{i j g}\right] \\
= & -\frac{1}{2} \boldsymbol{E}_{i}{ }^{T} \boldsymbol{Q}_{i} \boldsymbol{E}_{i}-E_{i}^{T} P_{i} B_{i}\left[\operatorname{sgn}\left(\boldsymbol{E}_{i}{ }^{T} \boldsymbol{P}_{i} \boldsymbol{B}_{i}\right) f_{i}^{V}-\Delta f_{s i}(\boldsymbol{x})\right] \\
& -\boldsymbol{E}_{i}{ }^{T} \boldsymbol{P}_{i} \boldsymbol{B}_{i} u_{H i} \sum_{j=1}^{3}\left[\operatorname{sgn}\left(\boldsymbol{E}_{i}{ }^{T} \boldsymbol{P}_{i} \boldsymbol{B}_{i} u_{a i}\right) g_{i j}^{V}-\Delta g_{i j g}(\boldsymbol{x})\right]- \\
& \varepsilon_{i f} \hat{m}_{i f}\left(\hat{m}_{i f}-m_{i f}\right)-\sum_{j=1}^{3} \varepsilon_{i j g} \hat{m}_{i j g}\left(\hat{m}_{i j g}-m_{i j g}\right)
\end{aligned}
$$


According to Assumptions 2, $\dot{V}_{H i}$ can be changed into,

$$
\begin{aligned}
\dot{V}_{H i} \leq & -\frac{1}{2} \boldsymbol{E}_{i}{ }^{T} \boldsymbol{Q}_{i} \boldsymbol{E}_{i}-\varepsilon_{i f} \hat{m}_{i f}\left(\hat{m}_{i f}-m_{i f}\right) \\
& -\sum_{j=1}^{3} \varepsilon_{i j g} \hat{m}_{i j g}\left(\hat{m}_{i j g}-m_{i j g}\right)
\end{aligned}
$$

By adopting the same inequality presented in (43), yields the following inequality,

$$
\begin{aligned}
\dot{V}_{H i} \leq & -\lambda_{\min }\left(\boldsymbol{Q}_{i}\right)\left\|\boldsymbol{E}_{i}\right\|^{2} / 2-\varepsilon_{i f} \hat{m}_{i f}\left(\hat{m}_{i f}-m_{i f}\right) \\
& -\sum_{j=1}^{3} \varepsilon_{i j g} \hat{m}_{i j g}\left(\hat{m}_{i j g}-m_{i j g}\right) \\
\leq & -\lambda_{\min }\left(\boldsymbol{Q}_{i}\right)\left\|\boldsymbol{E}_{i}\right\|^{2} / 2-\varepsilon_{i f}\left[\left(\hat{m}_{i f}+m_{i f} / 2\right)^{2}-\right. \\
& \left.m_{i f}^{2} / 4\right]-\sum_{j=1}^{3} \varepsilon_{i j g}\left[\left(\hat{m}_{i j g}+m_{i j g} / 2\right)^{2}-m_{i j g}^{2} / 4\right] \\
\leq & -\lambda_{\min }\left(\boldsymbol{Q}_{i}\right)\left\|\boldsymbol{E}_{i}\right\|^{2} / 2+\left[\varepsilon_{i f} m_{i f}^{2}+\sum_{j=1}^{3} \varepsilon_{i j g} m_{i j g}^{2}\right] / 4
\end{aligned}
$$

Similarly, $\dot{V}_{T i}<0$ holds for $\left\|\boldsymbol{E}_{i}\right\|>\left\{\left[2 \lambda_{\min }\left(\boldsymbol{Q}_{i}\right)\right]^{-1}\left(\varepsilon_{i f} m_{i f}^{2}+\right.\right.$ $\left.\left.\sum_{j=1}^{3} \varepsilon_{i j g} m_{i j g}^{2}\right)\right\}^{\frac{1}{2}}$ according to the inequality (60). Therefore, the tracking error vetor of attitude angle $\boldsymbol{E}_{i}$ converges to an arbitrarily small neighborhood around origin $D_{H E}$ given in (55) by tuning the designed parameters properly and $\boldsymbol{E}_{i} \in L_{\infty}$. On the basis of the definition of $\boldsymbol{E}_{i}$, it is obvious that $\boldsymbol{x} \in L_{\infty}$. From the estimation laws in (54), $\hat{\boldsymbol{m}}_{f}, \hat{\boldsymbol{m}}_{g} \in L_{\infty}$ hold as well. In the light of Assumption $1, \boldsymbol{f}(\boldsymbol{x}), \boldsymbol{g}(\boldsymbol{x}) \in L_{\infty}$ can be obtained. Moreover, considering $\ddot{\boldsymbol{y}}_{d} \in L_{\infty}$, it can be concluded that all items on the right side of Eq.(49) are bounded. Then, it is easy to know that $\boldsymbol{u}_{H} \in L_{\infty}$. Accordingly, we can draw the conclusion that the closed-loop control system of reentry RLV is semi-global asymptotic stable, and all related signals are both uniformly ultimately bounded.

Then the proof completes.

With the goal to guarantee the smooth input of the control actuator for reentry RLV, the overall control law is developed based on the two attitude controllers designed in Eqs.(24) and (49),

$\boldsymbol{u}=\tau \boldsymbol{u}_{H}+(1-\tau) \boldsymbol{u}_{T}$.

where $\tau$ denotes a weight coefficient, whicn is described as,

$\tau=\left\{\begin{array}{l}1, x \in \bar{\Omega} \\ \left(R_{x}-\|x\|\right) /\left(R_{x}-R_{0}\right), x \in \Omega-\Omega_{0} \\ 0, x \in \Omega_{0}\end{array}\right.$

460 where $\Omega_{0}=\left\{\boldsymbol{x}:\|\boldsymbol{x}\| \leq R_{0}\right\} \subset \Omega$, and $R_{0}$ is a finite constant pre-set by the designer.

Theorem 3: For the attitude control system (16) and (20) of reentry RLV, the overall attitude control law is designed as (61) together with the adaptive laws chosen as (37) and estimation 480 laws of boundary parameter vectors selected as (54), then the closed-loop control system can achieve semi-global asymptotic stable, and the attitude tracking error vector $\boldsymbol{E}_{i}$ converges to a small neighborhood $D_{E}$ around origin through tuning the controller parameters properly.

$$
\begin{gathered}
D_{E}=\left\{\boldsymbol{E}_{i}:\left\|\boldsymbol{E}_{i}\right\| \leq \frac{1}{2}\left\{[ \lambda _ { \operatorname { m i n } } ( \boldsymbol { Q } _ { i } ) ] ^ { - 1 } \left[2\left(\rho_{i} \bar{w}_{i}\right)^{2}\right.\right.\right. \\
\left.\left.\left.+\left(\varepsilon_{i f} m_{i f}^{2}+\sum_{j=1}^{3} \varepsilon_{i j g} m_{i j g}^{2}\right)\right]\right\}^{\frac{1}{2}}\right\}
\end{gathered}
$$

\section{Proof:}

Since the coefficients $\tau$ and $1-\tau$ are non-negative, thus chose the Lyapunov candidate function $V_{i}$ about the flight attitude dynamic as

$$
V_{i}=V_{T i}+V_{H i}
$$

According to the proofs in Theorem 1 and 2, we obtian the derivative of $V_{i}$ as

$$
\begin{aligned}
\dot{V}_{i}= & \frac{1}{2}\left[\left(\rho_{i} w_{i}^{*}\right)^{2}-\boldsymbol{E}_{i}^{T} \boldsymbol{Q}_{i} \boldsymbol{E}_{i}-\left(\frac{\boldsymbol{E}_{i}^{T} \boldsymbol{P}_{i} \boldsymbol{B}_{i}}{\rho_{i}}+\rho_{i} w_{i}^{*}\right)^{2}\right]-\gamma_{i \varepsilon} \alpha_{F i} \varepsilon_{F i}^{2} \\
- & \frac{1}{2} \boldsymbol{E}_{i}^{T} \boldsymbol{Q}_{i} \boldsymbol{E}_{i}-E_{i}^{T} P_{i} B_{i}\left[\operatorname{sgn}\left(\boldsymbol{E}_{i}^{T} \boldsymbol{P}_{i} \boldsymbol{B}_{i}\right) f_{i}^{V}-\Delta f_{s i}(\boldsymbol{x})\right]- \\
& \boldsymbol{E}_{i}^{T} \boldsymbol{P}_{i} \boldsymbol{B}_{i} u_{H i} \sum_{j=1}^{3}\left[\operatorname{sgn}\left(\boldsymbol{E}_{i}^{T} \boldsymbol{P}_{i} \boldsymbol{B}_{i} u_{a i}\right) g_{i j}^{V}-\Delta g_{i j g}(\boldsymbol{x})\right]- \\
& \varepsilon_{i f} \hat{m}_{i f}\left(\hat{m}_{i f}-m_{i f}\right)-\sum_{j=1}^{3} \varepsilon_{i j g} \hat{m}_{i j g}\left(\hat{m}_{i j g}-m_{i j g}\right)
\end{aligned}
$$

Based on the inequalities (45) and (60), yields $\dot{V}_{i}$

$\dot{V}_{i} \leq-\lambda_{\min }\left(\boldsymbol{Q}_{i}\right)\left\|\boldsymbol{E}_{i}\right\|^{2}+\left(\rho_{i} \bar{w}_{i}\right)^{2} / 2+\left[\varepsilon_{i f} m_{i f}^{2}+\sum_{j=1}^{3} \varepsilon_{i j g} m_{i j g}^{2}\right] / 4$

Thus, $\dot{V}_{i}<0$ holds for $\left\|\boldsymbol{E}_{i}\right\|>\frac{1}{2}\left\{\left[\lambda_{\min }\left(\boldsymbol{Q}_{i}\right)\right]^{-1}\left[2\left(\rho_{i} \bar{w}_{i}\right)^{2}+\right.\right.$ $\left.\left.\left(\varepsilon_{i f} m_{i f}^{2}+\sum_{j=1}^{3} \varepsilon_{i j g} m_{i j g}^{2}\right)\right]\right\}^{\frac{1}{2}}$ according to the inequality (66). According to the similar proofs in Theorem 1 and 2, we could conclude that the closed-loop control system of reentry RLV can achieve semi-global asymptotic stability under the overall attitude control law (61), and all related signals are both uniformly ultimately bounded.

Then the proof completes.

Remark 1: The adaptive laws in (40) and (41) combining with the Theorem 1 indicate that the boundedness of adjusting parameter vectors $\boldsymbol{\theta}_{i f}$ and $\boldsymbol{\theta}_{i j g}$ can be guaranteed by the developed control strategy. Similarly, the estimation laws in (54) with the Theorem 2 demonstrate that the boundedness of boundary parameter vectors $m_{i f}$ and $m_{i j g}$ can be guaranteed by the designed control scheme as well.

Remark 2: As can be seen from (44) and (60), the system stability and convergence rate depend on the appropriate selection of designed parameters. Generally, for the parameters $\gamma_{i f}, \gamma_{i j g}, \gamma_{i \varepsilon}$ in (37) and $\eta_{i f}, \eta_{i j g}$ in (54), larger values of these parameters would prompt the flight states of the RLV converge to the origin in a faster speed, while too large $\gamma_{i f}, \gamma_{i j g}, \gamma_{i \varepsilon}$ and 
$\eta_{i f}, \eta_{i j g}$ may result in unnecessary parameter chattering. Simiparameters would force the flight states converge to the origin more rapidly. However, too small $\varepsilon_{i f}, \varepsilon_{i j g}$ would lead to $\hat{m}_{i f}, \hat{m}_{i j g}$ too large, which is detrimental to the system stability.

Remark 3: From the flight control laws (24),(49) as well as the overall control law (61) of reentry RLV, it can be found that the proposed control approach does not need the boundary information of parameter uncertainties and external disturbances. The CAFHC scheme can adjust the designed parameters adaptively relying on the attitude tracking error vector $\boldsymbol{E}_{i}$ and filter modeling error $\varepsilon_{F i}$. Thus, it can guarantee that the attitude control system can realize a good tracking performance and have a good robustness performance simultaneously.

\section{Simulation results and discussions}

In this section, numerical simulations of attitude maneuver based on the 6-DoF dynamic model of reentry RLV are conducted to illustrate the performance and effectiveness of the attitude controller developed in the previous section.

\subsection{Simulation parameters setting}

The aircraft parameters adopted in the attitude controller design are presented in Table. 1, where the parameters are obtained by curve fitting approximations in MATLAB. In this simulation, the controlled variables are roll angle $\phi$, pitch angle $\theta$ and yaw angle $\psi$, respectively. The controller inputs are aileron deflection $\delta_{a}$, the elevator deflection $\delta_{e}$ and the rudder deflection Moreover, the parameter uncertainties and external disturbances are added to demonstrate the robustness of the proposed control scheme. And the uncertainty terms $\Delta f(x), \Delta g(x)$ and disturbance term $\boldsymbol{d}$ added to each channel of reentry RLV in the simulation are presented as,

$$
\begin{aligned}
& \Delta \boldsymbol{f}(\boldsymbol{x})=20 \% \boldsymbol{f}(\boldsymbol{x}), \Delta \boldsymbol{g}(\boldsymbol{x})=20 \% \boldsymbol{g}(\boldsymbol{x}) \\
& \boldsymbol{d}=\left[\begin{array}{l}
0.35+0.45 \sin (0.30 t) \\
2.25+0.75 \sin (0.50 t) \\
0.55+0.60 \sin (0.25 t)
\end{array}\right]
\end{aligned}
$$

Due to the fact that the flight states of RLVs vary with the flight height during its reentry process, thus two flight cases are taken into account in this paper to show the attitude tracking performance of the proposed control strategy more clearly. In case 1 , the initial altitude is $50 \mathrm{~km}$ and the expected outputs of $f_{535}$ angles are specified as sine functions. Although it is difficult to track the attitude angles for reentry RLV in accordance with the command of sine function in practical flight process, it is feasible to adopt the sine function as expected command in order to better verify the effectiveness of the developed attitude ${ }_{540}$ roller in simulation. In case 2 , the initial altitude is $30 \mathrm{~km}$ and the expected outputs are chosen as sine functions as well.

Furthermore, in the design of fuzzy logic system (FLS), the membership function of $x_{i}$ is selected as Gaussian type membership function [31], and the corresponding controller param-545 where the parameter $r_{i}=2 \rho_{i}^{2}$, and $\rho_{1}=\rho_{2}=\rho_{3}=0.2$.

Table 1: Detailed values of aircraft parameters

\begin{tabular}{cccc}
\hline Terms & Values & Terms & Values \\
\hline$m$ & $7500 \mathrm{~kg}$ & $C_{Y}^{\beta}$ & $4.63 \mathrm{e}-04$ \\
$\bar{b}$ & $3.12 \mathrm{~m}$ & $C_{Y}^{\delta_{r}}$ & 0.0039 \\
$\bar{c}$ & $1.30 \mathrm{~m}$ & $C_{l}^{\beta}$ & $9.80 \mathrm{e}-28$ \\
$S$ & $4.17 \mathrm{~m}^{2}$ & $C_{l}^{\delta_{a}}$ & 0.0023 \\
$J_{x x}$ & $885 \mathrm{~kg} \cdot \mathrm{m}^{2}$ & $C_{l}^{\delta_{r}}$ & 0.0028 \\
$J_{y y}$ & $8110 \mathrm{~kg} \cdot \mathrm{m}^{2}$ & $C_{l}^{r}$ & 0.2201 \\
$J_{z z}$ & $7770 \mathrm{~kg} \cdot \mathrm{m}^{2}$ & $C_{m}^{0}$ & -0.0151 \\
$J_{x z}$ & $-17.40 \mathrm{~kg} \cdot \mathrm{m}^{2}$ & $C_{m}^{\alpha}$ & 0.0117 \\
$C_{L}^{0}$ & -0.404 & $C_{m}^{\beta}$ & $1.134 \mathrm{e}-10$ \\
$C_{L}^{\alpha}$ & 0.0699 & $C_{m}^{\delta_{e}}$ & -0.008 \\
$C_{L}^{\delta_{e}}$ & 0.0026 & $C_{m}^{q}$ & 0.417 \\
$C_{D}^{0}$ & -0.239 & $C_{n}^{\beta}$ & $7.95 \mathrm{e}-04$ \\
$C_{D}^{\alpha}$ & 0.035 & $C_{n}^{\delta_{a}}$ & $4.997 \mathrm{e}-04$ \\
$C_{D}^{\beta}$ & $-8.30 \mathrm{e}-10$ & $C_{n}^{\delta_{r}}$ & 0.0041 \\
$C_{D}^{\delta_{e}}$ & 0.0021 & $C_{n}^{p}$ & 0.142 \\
\hline & & &
\end{tabular}

Table 2: Controller parameters of the proposed strategy

\begin{tabular}{cccc}
\hline Parameters & Values & Parameters & Values \\
\hline$k_{11}, k_{12}$ & 5,40 & $\gamma_{g}$ & $95 * \boldsymbol{I}_{3 \times 3}$ \\
$k_{21}, k_{22}$ & 5,25 & $\boldsymbol{\eta}_{g}$ & $90 * \boldsymbol{I}_{3 \times 3}$ \\
$k_{31}, k_{32}$ & 5,30 & $\boldsymbol{\varepsilon}_{g}$ & $0.1 * \boldsymbol{I}_{3 \times 3}$ \\
$\beta_{1}, \beta_{2}, \beta_{3}$ & $1,1,1$ & $\gamma_{1 \varepsilon}, \gamma_{1 \varepsilon}, \gamma_{1 \varepsilon}$ & $0.12,0.12,0.12$ \\
$\alpha_{F 1}, \alpha_{F 2}, \alpha_{F 3}$ & $12,10,12$ & $\eta_{1 f}, \eta_{2 f}, \eta_{3 f}$ & $90,85,90$ \\
$\gamma_{1 f}, \gamma_{2 f}, \gamma_{3 f}$ & $100,100,90$ & $\varepsilon_{1 f}, \varepsilon_{2 f}, \varepsilon_{3 f}$ & $0.12,0.12,0.12$ \\
\hline
\end{tabular}

\subsection{Simulation discussions}

Case 1: In this flight condition, the initial flight altitude and velocity are chosen as $50 \mathrm{~km}$ and $15 \mathrm{Mach}$ respectively. And the initial aerodynamic angle $\beta$, attitude angles $\phi, \psi$ and angular rates are all set to be zeros, and $\alpha=\theta=25 \mathrm{deg}$. Furthermore, all the initial actuator deflections $\delta_{a}, \delta_{e}, \delta_{r}$ are set to be zeros as well, and choose $R_{x}=19, R_{0}=13$. The simulation results for this case are presented in Fig. 3-Fig. 5, and some necessary curve explanations are shown in the figures as well for the better demonstration of flight process. In addition, an adaptive fuzzy $\mathrm{H}$-infinity control (AFHC) method is chose to compare the attitude tracking performance of the vehicle.

The curves of roll angle, pitch angle and yaw angle tracking for the RLV are provided in Fig. 3 (a), (b) and (c) respectively, where the red solid line denotes the desired tracking commands, the blue solid line denotes the actual output signal of attitude an- 
gle under the CAFHC strategy and the green solid line denotes the actual output under the AFHC strategy. It can be seen from the figures that the attitude angles are able to track the desired commands stably and smoothly in the presence of the param-570 eter uncertainties and external disturbances during its reentry phase. In addition, the attitude control scheme designed in this paper performs better than the AFHC strategy.
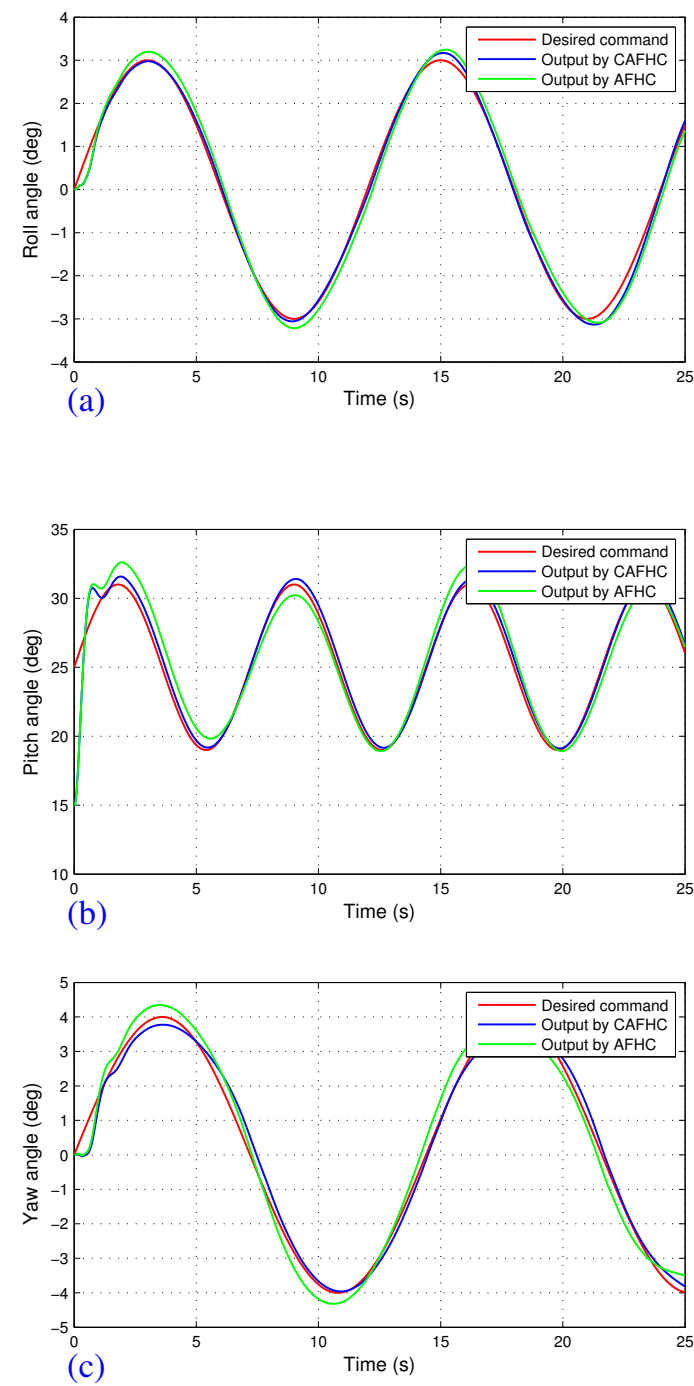

Fig. 3. Curve of RLV attitude angle tracking in case 1.

(a)Roll angle;(b)Pitch angle;(c)Yaw angle

To verify the tracking performance of attitude angle more clearly, the curves of roll angle, pitch angle and yaw angle tracking error are presented in Fig. 4 (a), (b) and (c). From the simulation results, it is obvious that the attitude controller developed in this study can track the desired commands within a short period of time. Since the airborne condition and flight state in the height of $50 \mathrm{~km}$ are more complicated, thus there is about $10 \mathrm{deg}$ changing magnitude within 0.25 seconds as580 showed in the curve of pitch angle tracking error. And then the tracking error of three-channel attitude angles converge to a ing errors by the CAFHC method are smaller than by the AFHC method as shown in the figures.

In the meantime, the time histories of the deflections of control actuators are given in Fig. 5 (a), (b) and (c). From the simulation, we can observe that the control actuators vary within a reasonable range (i.e., all the control actuators' deflections vary between -20deg and $20 \mathrm{deg}$ ), which ensures that the attitude control system has a good tracking performance. As the "couangle channel, so the responses of aileron deflection and rudder deflection show some small fluctuations, while these fluctuations are within the control range and they do not affect the attitude tracking for reentry RLV.
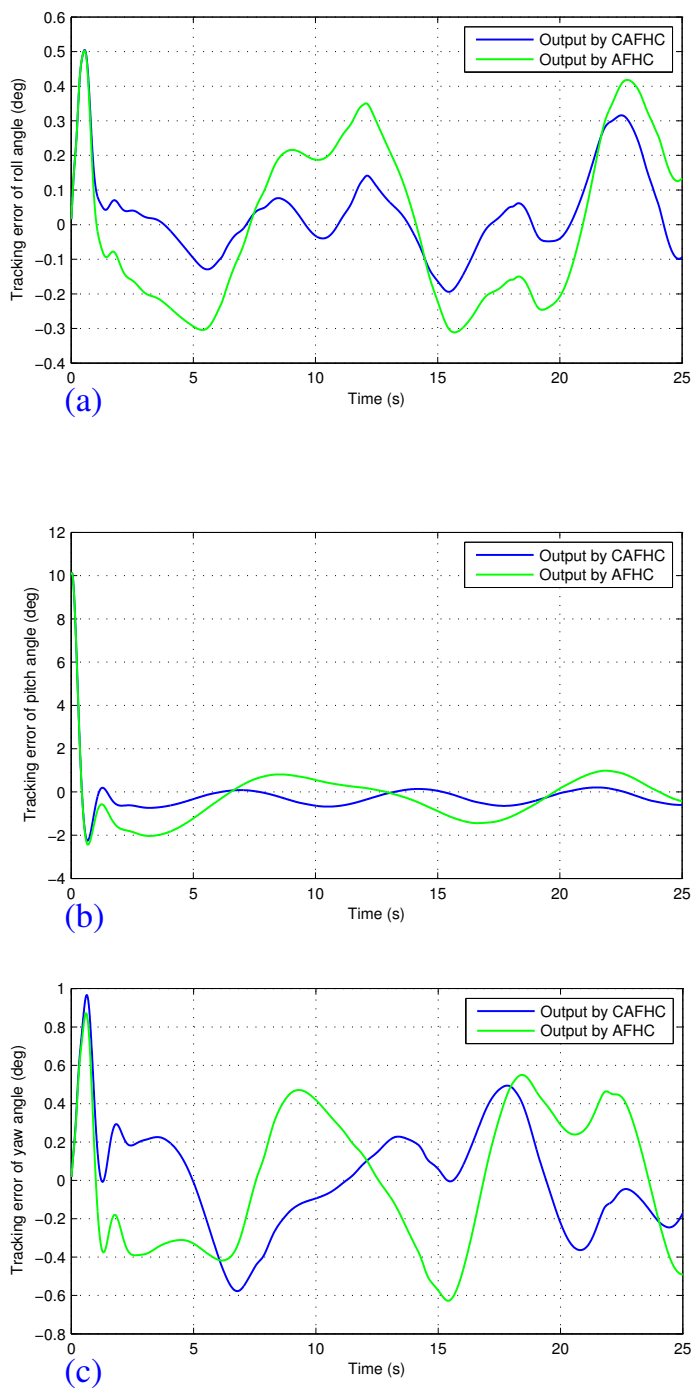

Fig. 4. Curve of attitude angle tracking error in case 1.

(a)Roll angle tracking error;(b)Pitch angle tracking error;(c)Yaw angle tracking error

Case 2: In this case, the initial flight altitude and velocity are selected as $30 \mathrm{~km}$ and 8 Mach respectively. Since the atmospheric density at this altitude is thicker than the atmospheric density at $50 \mathrm{~km}$, hence it unnecessary to use the high angle of attack and pitch for control input in this simulation. Then 
$\theta=15 \mathrm{deg}$, and the initial values of the other parameters setting of the RLV are set as same as the simulation in case 1. And choose $R_{x}=12, R_{0}=10$. The simulation results are provided in Fig. 6-Fig. 8, and several curve explanations are marked in the figures as well for the better illustration of dymic process. Besides, the attitude tracking performance under the AFHC strategy is compared with the proposed scheme in this paper.

The flight attitude tracking curve of the RLV during the reentry phase is provided in Fig. 6, where the red solid line denotes the desired tracking commands, the blue solid line denotes the actual output of attitude angle by the CAFHC strategy and the green solid line denotes the actual output by the AFHC strategy. As shown in Fig. 6, it is obvious that the roll angle, pitch angle and yaw angle can track the desired commands well under the parameter uncertainties and external disturbances in this flight condition as well, and the actual attitude angle of reentry RLV by the CAFHC scheme can respond to the guidance commands with a higher accuracy than by the AFHC scheme.


Fig. 5. Response curve of RLV control actuator in case 1. (a)Aileron deflection;(b)Elevator deflection;(c)Rudder deflection
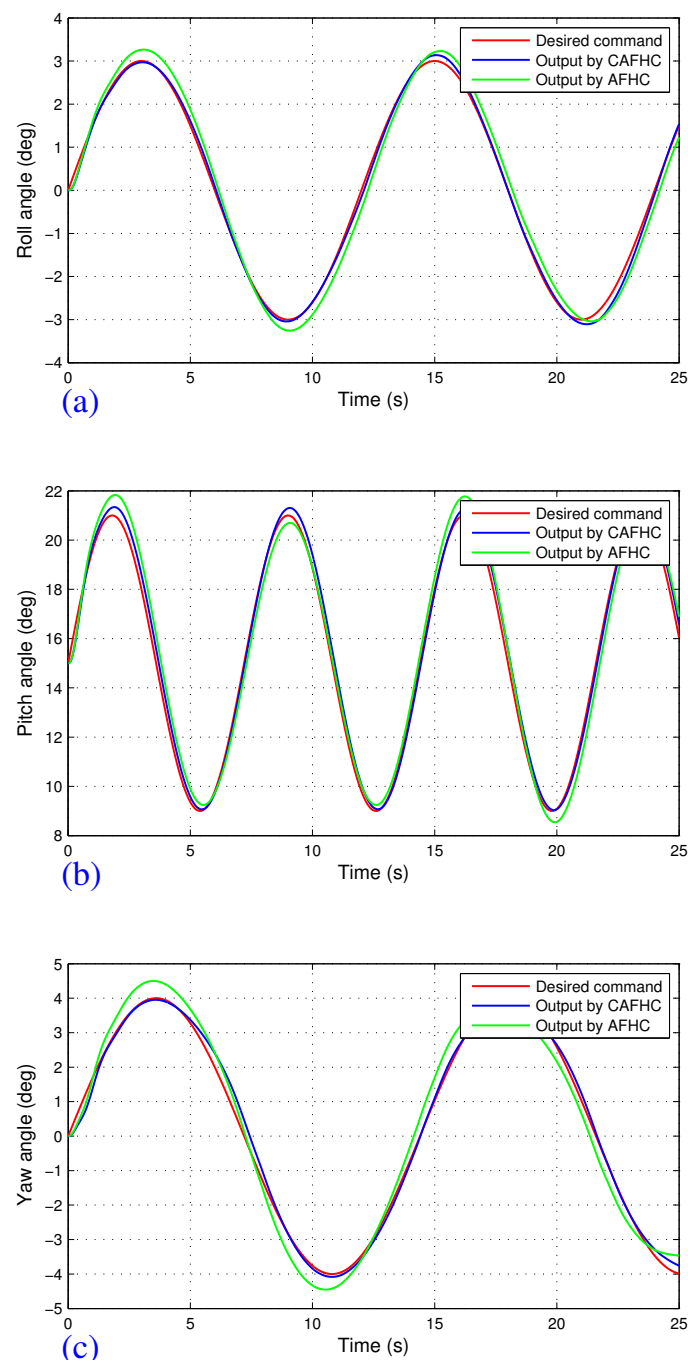

Fig. 6. Curve of RLV attitude angle tracking in case 2 . (a)Roll angle;(b)Pitch angle;(c)Yaw angle

The tracking errors of attitude angulars are shown in Fig. 7 (a), (b) and (c) respectively, which implies that the reentry vehicle can realize a stable tracking in a short time under the action of attitude control law proposed in this paper. And the maximum tracking error of roll angle, pitch angle and yaw angle does not exceed $0.65 \mathrm{deg}$, which demonstrates that the developed attitude controller in this paper not only tracks the guidance commands with a high accuracy, but also outperforms the AFHC approach.

Meanwhile, with the aim to verify the validity and capability of the designed attitude controller, the time histories of control actuators are presented in Fig. 8. From the simulation results, we can come to a conclusion that the deflections of control actuators response timely in the permitted range as the guidance commands change.

\section{Conclusion}

In this paper, a novel attitude control scheme based on CAFHC is developed to achieve a stable and reliable flight process for RLVs during reentry phase where the parameter un- 


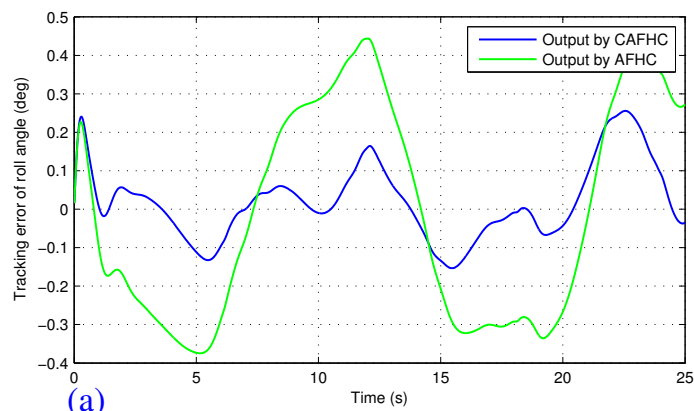

(a)
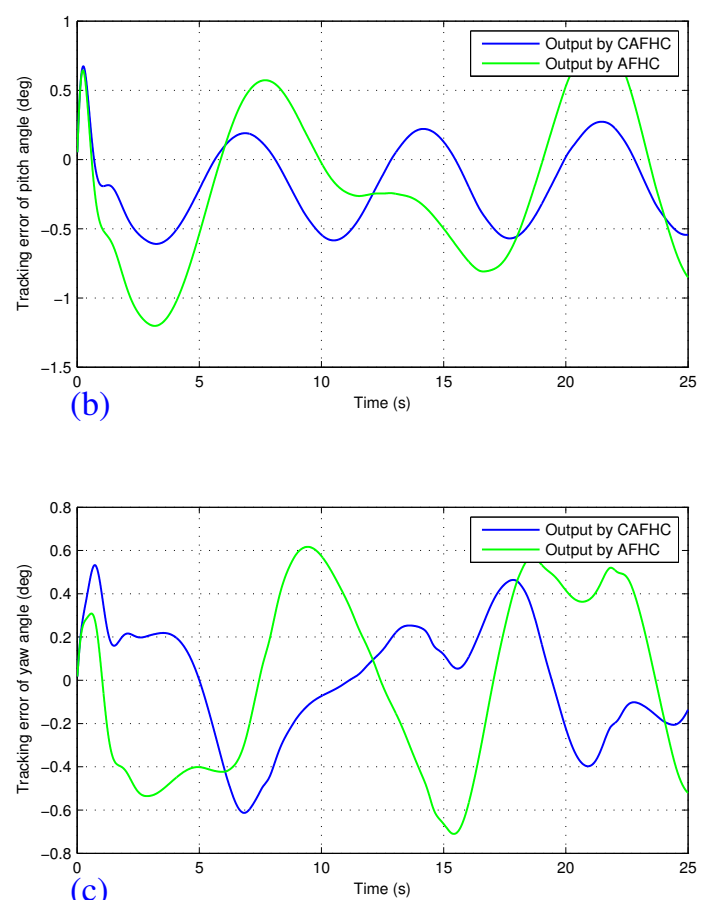

Fig. 7. Curve of attitude angle tracking error in case 2.

(a)Roll angle tracking error;(b)Pitch angle tracking error;(c)Yaw angle tracking error

certainties and unknown external disturbances are taken into consideration. The design principle of the proposed control strategy can be summarized by three steps as follows. First, the attitude tracking controller is designed based on adaptive fuzzy $\mathrm{H}$-infinity control method to approximate the system uncertainty terms and improve the tracking performance within the fuzzy approximation region of flight attitude. Second, the attitude stabilization controller using boundary adaptive technique is developed to guarantee the system robustness and the boundness of approximation error outside the fuzzy approximation region of flight attitude. Moreover, to obtain a smooth input of the control actuator for the RLV, the overall controller ${ }_{650}$ is employed on the basis of the above two attitude controllers. In addition, the stability of the closed-loop system can be guaranteed through Lyapunov stability analysis. Finally, the 6-DoF dynamic flight simulation results are provided to demonstrate that the effectiveness and control performance of the designed of parameter uncertainties and external disturbances. Meanwhile, the simulation results and analysis have illustrated that the proposed control strategy performs better and track the desired attitude angles for reentry RLV with a higher accuracy 645 than the AFHC method.
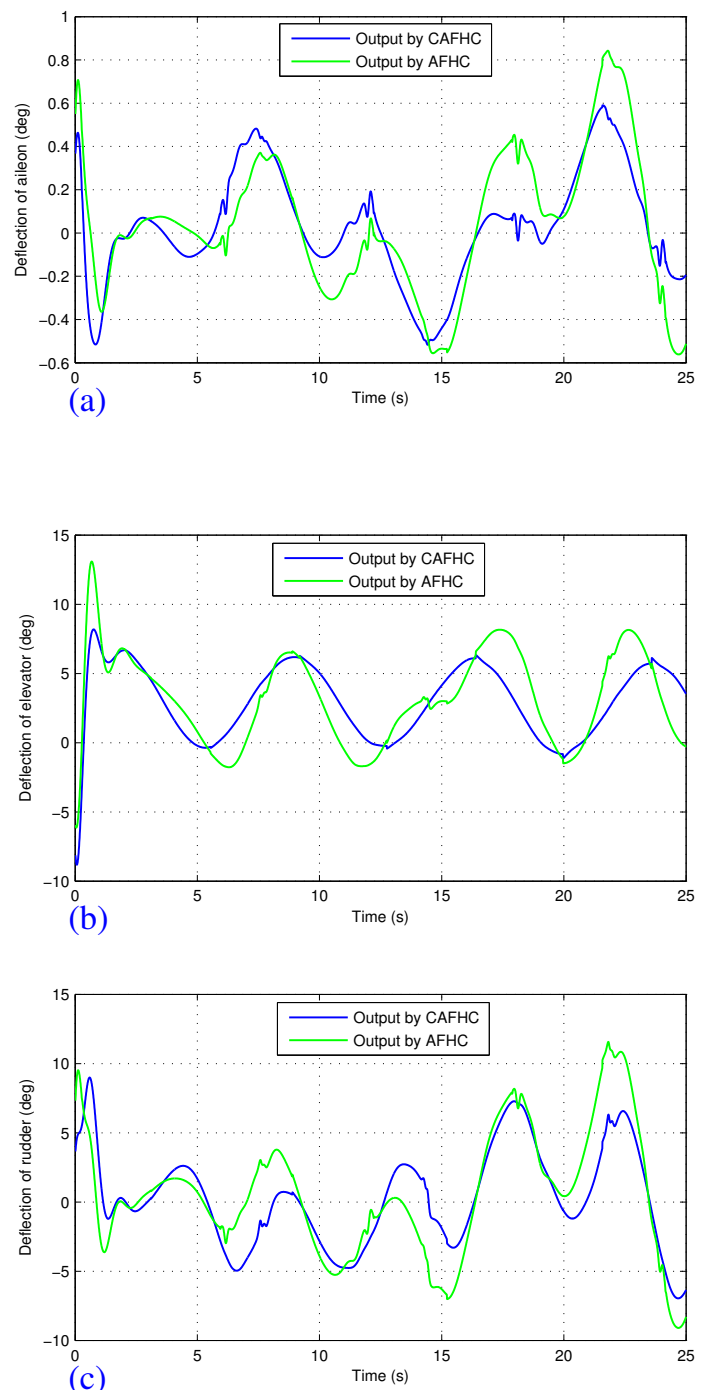

Fig. 8. Response curve of RLV control actuator in case 2.

(a)Aileron deflection;(b)Elevator deflection;(c)Rudder deflection

\section{Acknowledgments}

This works was partially supported by the Natural Science Foundation of China (NSFC) under Grant (61273092, 61673294, 91016018) and the Natural Science Foundation of Tianjin under Grant 12JCZDJC30300.

\section{References}

[1] S. Mathavaraj, O. Halbe, R. Padhi, Robust control of a reusable launch vehicle in reentry phase using model following neuro-adaptive design, in: AIAA guidance, navigation, and control conference, 2010, p. 8312. 
[2] B. Tian, W. Fan, R. Su, Q. Zong, Real-time trajectory and attitude coordination control for reusable launch vehicle in reentry phase, IEEE Transactions on Industrial Electronics 62 (3) (2015) 1639-1650.

[3] Q. Zong, F. Wang, B. Tian, R. Su, Robust adaptive dynamic surface control design for a flexible air-breathing hypersonic vehicle with input con-730 straints and uncertainty, Nonlinear Dynamics 78 (1) (2014) 289-315.

[4] N. Berend, C. Talbot, Overview of some optimal control methods adapted to expendable and reusable launch vehicle trajectories, Aerospace science and technology 10 (3) (2006) 222-232.

[5] Z. Gao, B. Jiang, P. Shi, M. Qian, J. Lin, Active fault tolerant control735 design for reusable launch vehicle using adaptive sliding mode technique, Journal of the Franklin Institute 349 (4) (2012) 1543-1560.

[6] R. Su, Q. Zong, B. Tian, M. You, Comprehensive design of disturbance observer and non-singular terminal sliding mode control for reusable launch vehicles, IET Control Theory \& Applications 9 (12) (2015) 1821-740 1830 .

[7] Z. Wang, Z. Wu, Y. Du, Robust adaptive backstepping control for reentry reusable launch vehicles, Acta Astronautica 126 (2016) 258-264.

[8] O. Halbe, R. G. Raja, R. Padhi, Robust reentry guidance of a reusable launch vehicle using model predictive static programming, Journal of 745 Guidance, Control, and Dynamics 37 (1) (2013) 134-148.

[9] T. Dwyer, Exact nonlinear control of large angle rotational maneuvers, IEEE Transactions on Automatic Control 29 (9) (1984) 769-774.

[10] G. Dukeman, Profile-following entry guidance using linear quadratic regulator theory, in: AIAA Guidance, Navigation, and Control Conference 750 and Exhibit, 2002.

[11] M. Kerr, A. Marcos, L. Peñin, E. Bornschlegl, Gain scheduled fdi for a re-entry vehicle, in: AIAA Guidance, Navigation and Control Conference and Exhibit, 2008, p. 7266.

[12] Y. Huang, C. Sun, C. Qian, L. Wang, Slow-fast loop gain-scheduled755 switching attitude tracking control for a near-space hypersonic vehicle, Proceedings of the Institution of Mechanical Engineers, Part I: Journal of Systems and Control Engineering 227 (1) (2013) 96-109.

[13] X. Huang, Q. Wang, Y. Wang, Y. Hou, C. Dong, Adaptive augmentation of gain-scheduled controller for aerospace vehicles, Journal of Systems760 Engineering and Electronics 24 (2) (2013) 272-280.

[14] J. Recasens, Q. Chu, J. Mulder, Robust model predictive control of a feedback linearized system for a lifting-body re-entry vehicle, in: AIAA guidance, navigation, and control conference and exhibit, 2005, p. 6147.

[15] X.-L. Su, J.-Q. Yu, Y.-F. Wang, L.-1. Wang, Moving mass actuated reentry765 vehicle control based on trajectory linearization, International Journal of Aeronautical and Space Sciences 14 (3) (2013) 247-255

[16] S. Xingling, W. Honglun, Sliding mode based trajectory linearization control for hypersonic reentry vehicle via extended disturbance observer, ISA transactions 53 (6) (2014) 1771-1786.

700 [17] X. Shao, H. Wang, Active disturbance rejection based trajectory linearization control for hypersonic reentry vehicle with bounded uncertainties, ISA transactions 54 (2015) 27-38.

[18] R. Da Costa, Q. Chu, J. Mulder, Reentry flight controller design using nonlinear dynamic inversion, Journal of Spacecraft and Rockets 40 (1)775 (2003) 64-71.

[19] S. Sieberling, Q. Chu, J. Mulder, Robust flight control using incremental nonlinear dynamic inversion and angular acceleration prediction, Journal of Guidance, Control, and Dynamics 33 (6) (2010) 1732-1742.

[20] U. Ansari, A. H. Bajodah, Generalized dynamic inversion scheme for 780 satellite launch vehicle attitude control, IFAC-PapersOnLine 48 (9) (2015) 114-119.

[21] Q. Lam, P. Krishnamurthy, F. Khorrami, Enhancing flight control system performance using sdre based controller as an augmentation layer, in: AIAA guidance, navigation, and control conference, 2009, p. 6171. 78

[22] J. Farrell, M. Polycarpou, M. Sharma, Adaptive backstepping with magnitude, rate, and bandwidth constraints: aircraft longitude control, in: American Control Conference, 2003. Proceedings of the, 2003, pp. 38983904 vol.5.

[23] J. Farrell, M. Sharma, M. Polycarpou, Backstepping-based flight control790 with adaptive function approximation, Journal of Guidance, Control, and Dynamics 28 (6) (2005) 1089-1102.

[24] C. E. Hall, Y. B. Shtessel, Sliding mode disturbance observer-based control for a reusable launch vehicle, Journal of guidance, control, and dynamics 29 (6) (2006) 1315-1328.

725 [25] J. E. Stott, Y. B. Shtessel, Launch vehicle attitude control using sliding mode control and observation techniques, Journal of the Franklin Institute 349 (2) (2012) 397-412.

[26] H. Prathap, V. Brinda, S. Ushakumari, Robust flight control of a typical rlv during re-entry phase, in: Control Applications (CCA), 2013 IEEE International Conference on, IEEE, 2013, pp. 716-721.

[27] F. Wang, C. Hua, Q. Zong, Attitude control of reusable launch vehicle in reentry phase with input constraint via robust adaptive backstepping control, International Journal of Adaptive Control and Signal Processing 29 (10) (2015) 1308-1327.

[28] G. Jee, A. Yalagach, V. Brinda, V. Lalithambika, M. Dhekane, Controller design for lateral dynamics of reusable launch vehicle by h-infinity mixed sensitivity approach, IFAC Proceedings Volumes 47 (1) (2014) 171-175.

[29] J. Xiao-Zheng, Y. Guang-Hong, Robust adaptive fault-tolerant compensation control with actuator failures and bounded disturbances, Acta $\mathrm{Au}$ tomatica Sinica 35 (3) (2009) 305-309.

[30] L. Fiorentini, A. Serrani, M. A. Bolender, D. B. Doman, Nonlinear robust adaptive control of flexible air-breathing hypersonic vehicles, Journal of guidance, control, and dynamics 32 (2) (2009) 402-417.

[31] D. Gao, Z. Sun, T. Du, Dynamic surface control for hypersonic aircraft using fuzzy logic system, in: Automation and Logistics, 2007 IEEE International Conference on, IEEE, 2007, pp. 2314-2319.

[32] C. Min, D. Lee, K. Cho, S. Jo, J. Yang, W. Lee, Control of approach and landing phase for reentry vehicle using fuzzy logic, Aerospace Science and Technology 15 (4) (2011) 269-282.

[33] L. Cervantes, O. Castillo, P. Melin, Intelligent control of nonlinear dynamic plants using a hierarchical modular approach and type-2 fuzzy logic, Advances in Soft Computing (2011) 1-12.

[34] X. Luo, J. Li, Fuzzy dynamic characteristic model based attitude control of hypersonic vehicle in gliding phase, Science China Information Sciences 54 (3) (2011) 448-459.

[35] B. Jiang, Z. Gao, P. Shi, Y. Xu, Adaptive fault-tolerant tracking control of near-space vehicle using takagi-sugeno fuzzy models, IEEE Transactions on Fuzzy Systems 18 (5) (2010) 1000-1007.

[36] H. Huang, Z. Zhang, Characteristic model-based $h 2 / \mathrm{h} \infty$ robust adaptive control during the re-entry of hypersonic cruise vehicles, Science China Information Sciences 58 (1) (2015) 1-21.

[37] Q. Shen, B. Jiang, V. Cocquempot, Fuzzy logic system-based adaptive fault-tolerant control for near-space vehicle attitude dynamics with actuator faults, IEEE Transactions on Fuzzy Systems 21 (2) (2013) 289-300.

[38] S. Tong, X. He, Y. Li, H. Zhang, Adaptive fuzzy backstepping robust control for uncertain nonlinear systems based on small-gain approach, Fuzzy Sets and Systems 161 (6) (2010) 771-796.

[39] T.-S. Wu, M. Karkoub, H.-S. Chen, W.-S. Yu, M.-G. Her, Robust tracking observer-based adaptive fuzzy control design for uncertain nonlinear mimo systems with time delayed states, Information Sciences 290 (2015) 86-105.

[40] H. F. Ghavidel, A. A. Kalat, Robust control for mimo hybrid dynamical system of underwater vehicles by composite adaptive fuzzy estimation of uncertainties, Nonlinear Dynamics (2017) 1-19.

[41] S. A. Snell, D. F. Nns, W. L. Arrard, Nonlinear inversion flight control for a supermaneuverable aircraft, Journal of Guidance Control and Dynamics 15 (4) (1992) 976-984.

[42] J. J. Recasens, Q. P. Chu, J. A. Mulder, Robust model predictive control of a feedback linearized system for a lifting-body re-entry vehicle, in: AIAA Guidance, Navigation, and Control Conference and Exhibit, AIAA, 2005, p. 6147.

[43] W. Gai, H. Wang, J. Zhang, Y. Li, Adaptive neural network dynamic inversion with prescribed performance for aircraft flight control, Journal of Applied Mathematics 2013.

[44] L.-X. Wang, Design and analysis of fuzzy identifiers of nonlinear dynamic systems, IEEE transactions on automatic control 40 (1) (1995) 1123.

[45] S. S. Ge, C. C. Hang, T. H. Lee, T. Zhang, Stable adaptive neural network control, Vol. 13, Springer Science \& Business Media, 2013.

[46] P. A. Phan, T. Gale, Two-mode adaptive fuzzy control with approximation error estimator, IEEE Transactions on Fuzzy Systems 15 (5) (2007) 943955. 